\title{
Audit of High-Energy Photon Beams in Belgian and Dutch Radiotherapy Departments
}

\author{
NEDERLANDSE COMMISSIE VOOR STRALINGSDOSIMETRIE
}

Report 23 of the Netherlands Commission on Radiation Dosimetry

December 2013

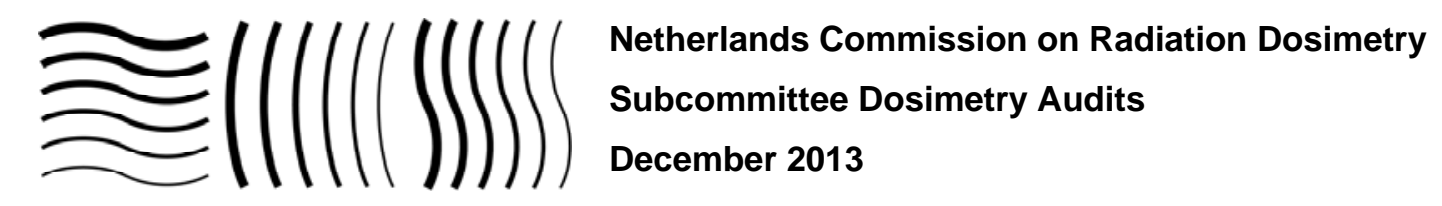


https://doi.org/10.25030/ncs-023 The NCS report has been downloaded on 26 Apr 2023 


\title{
Audit of High-Energy Photon Beams in Belgian and Dutch Radiotherapy Departments
}

\author{
NEDERLANDSE COMMISSIE VOOR STRALINGSDOSIMETRIE \\ Report 23 of the Netherlands Commission on Radiation Dosimetry
}

Authors

T.J. Perik

L.A. de Prez

A.H.L. Aalbers

A. Monseux

F. Sergent

F.W. Wittkämper

J.B. van de Kamer

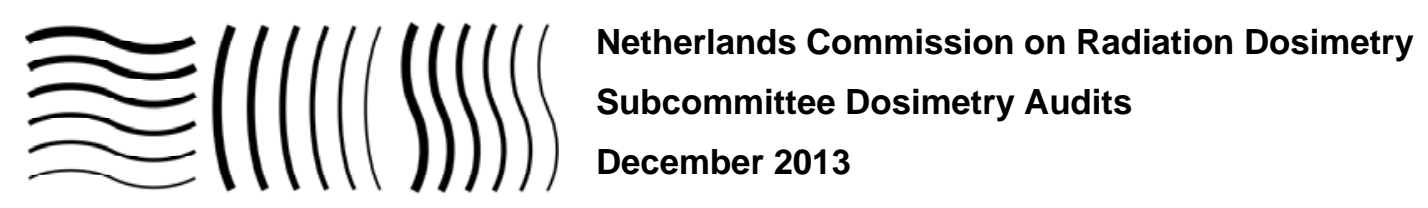




\section{Disclaimer regarding NCS reports}

The NCS frequently publishes reports for fellow professionals in which recommendations are given for various quality control procedures or otherwise. The members of the NCS board and the members of the concerning subcommittee do not claim any authority exceeding that of their professional expertise. Responsibility on how the NCS recommendations are implemented lies with the user, taking into account the practice in his/her institution. 


\section{Preface}

The Nederlandse Commissie voor Stralingsdosimetrie (NCS, Netherlands Commission on Radiation Dosimetry, http://www.radiationdosimetry.org) was officially established on 3 September 1982 with the aim of promoting the appropriate use of dosimetry of ionising radiation both for scientific research and practical applications. The NCS is chaired by a board of scientists, installed upon the suggestion of the supporting societies, including the Nederlandse Vereniging voor Radiotherapie en Oncologie (Netherlands Society for Radiotherapy and Oncology), the Nederlandse Vereniging voor Nucleaire Geneeskunde (Dutch Society of Nuclear Medicine), the Nederlandse Vereniging voor Klinische Fysica (Dutch Society for Medical Physics), the Nederlandse Vereniging voor Radiobiologie (Netherlands Radiobiological Society), the Nederlandse Vereniging voor Stralingshygiëne (Netherlands Society for Radiological Protection), the Nederlandse Vereniging voor Medische Beeldvorming en Radiotherapie (Dutch Society for Medical Imaging and Radiotherapy), the Nederlandse Vereniging van Klinisch Fysisch Medewerkers (Dutch Society for Medical Physics Engineers), the Nederlandse Vereniging voor Radiologie (Radiological Society of the Netherlands) and the Belgische Vereniging voor Ziekenhuisfysici/Société Belge des Physiciens des Hôpitaux (Belgian Hospital Physicists Association). To pursue its aims, the NCS accomplishes the following tasks: participation in dosimetry standardisation and promotion of dosimetry intercomparisons, drafting of dosimetry protocols, collection and evaluation of physical data related to dosimetry. Furthermore, the commission shall maintain or establish links with national and international organisations concerned with ionising radiation and promulgate information on new developments in the field of radiation dosimetry.

Current members of the board of the NCS

J.B. van de Kamer, Chairman T.W.M. Grimbergen, Vice-Chairman J. de Pooter, Secretary J.M.J. Hermans, Treasurer A. Van Der Plaetsen

A. Spilt F.W. Wittkämper M.K. Zeeman J.R. de Jong P. Sminia K. Franken 
https://doi.org/10.25030/ncs-023 The NCS report has been downloaded on 26 Apr 2023 


\section{Audit of High-Energy Photon Beams in Belgian and Dutch Radiotherapy Departments}

Members of the subcommittee:

T.J. Perik (chairman) ${ }^{1}$

L.A. de Prez (vice-chairman) ${ }^{2}$

M. Dwarswaard (secretary) ${ }^{3}$

J. Martens (treasurer) ${ }^{4}$

S. van het Schip (logistics) ${ }^{5}$

A.H.L. Aalbers (advisor) ${ }^{2}$

K. Feyen ${ }^{6}$

E. Loeff ${ }^{7}$

J. Hermans ${ }^{4}$

A. Monseux ${ }^{8}$

E. Peeters-Cleven ${ }^{9}$

N. Planteydt ${ }^{10}$

F. Sergent ${ }^{11}$

F.W. Wittkämper (advisor) ${ }^{1}$

${ }^{1}$ The Netherlands Cancer Institute, Amsterdam, the Netherlands

${ }^{2} \mathrm{VSL}$ - Dutch Metrology Institute, Delft, the Netherlands

${ }^{3} \mathrm{MCA}$, Alkmaar, the Netherlands

${ }^{4}$ MAASTRO Clinic, Maastricht, the Netherlands

${ }^{5}$ Catharina Ziekenhuis, Eindhoven, the Netherlands

${ }^{6}$ AZ St. Maarten, Duffel, Belgium

${ }^{7}$ Erasmus MC, Rotterdam, the Netherlands

${ }^{8} \mathrm{CHU}$, Montigny-le-Tilleul, Belgium

${ }^{9} \mathrm{MST}$, Enschede, the Netherlands

${ }^{10} \mathrm{ZRTI}$, Vlissingen, the Netherlands

${ }^{11} \mathrm{CMSE}$, Namur, Belgium

NCS, Delft, the Netherlands

For more information on NCS Reports, see http://radiationdosimetry.org 
https://doi.org/10.25030/ncs-023 The NCS report has been downloaded on 26 Apr 2023 


\section{Summary}

A dosimetric intercomparison has been carried out in 26 radiotherapy departments in Belgium and the Netherlands during 2009 and 2010. Dose measurements were performed in order to evaluate the uniformity in dosimetry outcome and as an external quality control regarding the implementation of the NCS-18 Code of Practice (CoP). This intercomparison was carried out on a single treatment unit in each centre. All measurements were performed in water with an ionisation chamber following the NCS-18 CoP [1]. At the time of the audit, 17 centres implemented, or were able to use, the NCS-18 CoP.

On average the deviations were $-0.3 \%$ (maximum $-1.4 \%$ ), comparing the institutes measurement and those of the audit team for the NCS $18 \mathrm{CoP}$. Based on these results we conclude that the implementation of the NCS-18 CoP for photon beams did not introduce significant deviations in dosimetry. 
https://doi.org/10.25030/ncs-023 The NCS report has been downloaded on 26 Apr 2023 


\section{Contents}

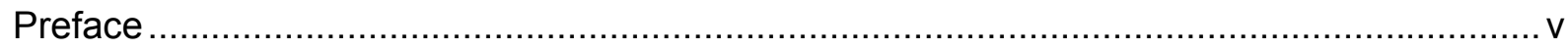

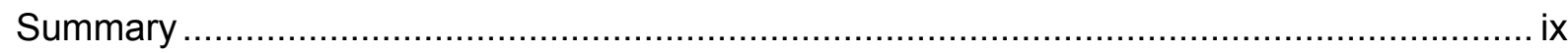

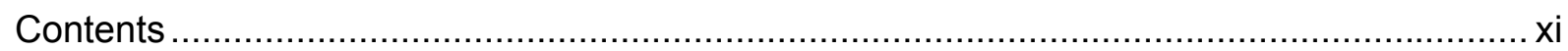

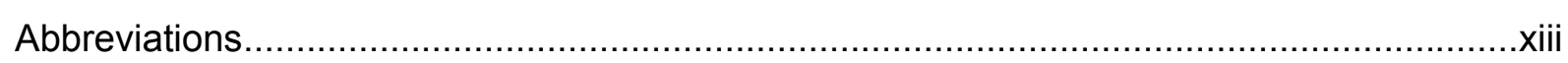

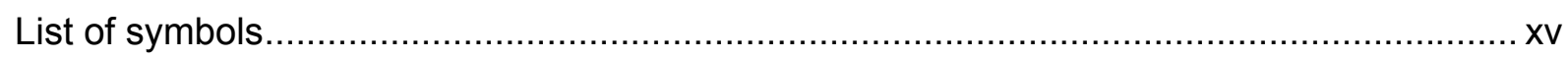

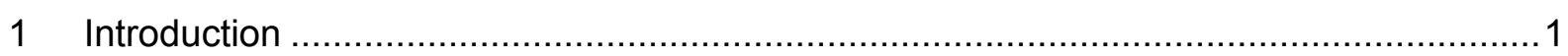

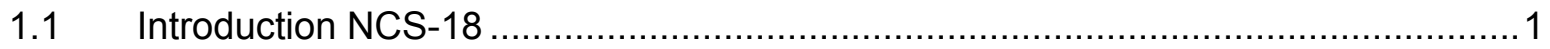

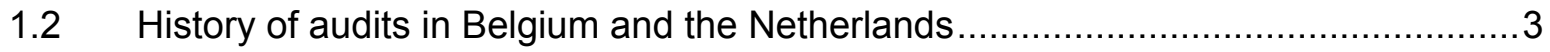

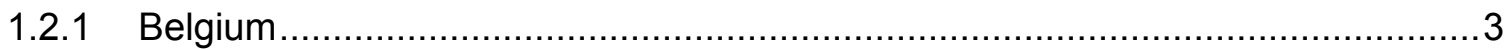

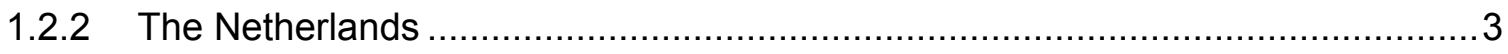

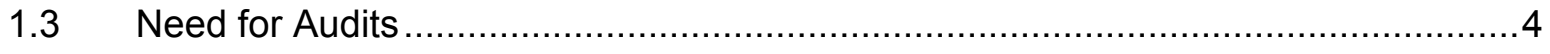

1.3.1 Establishment of the NCS subcommittee Dosimetry Audits ..............................4

1.3.2 Goal of the NCS subcommittee Dosimetry Audits ......................................... 5

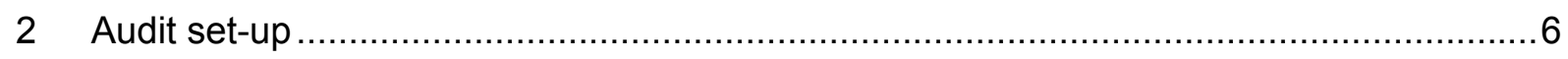

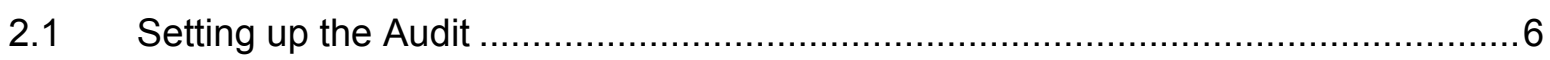

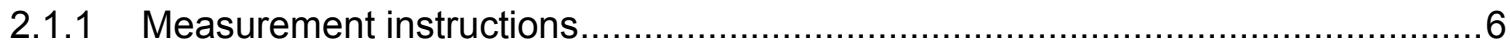

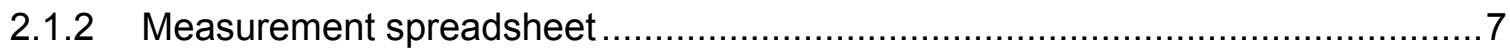

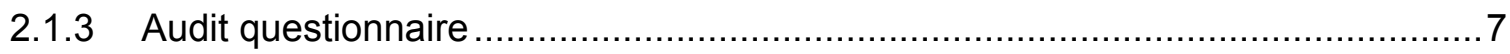

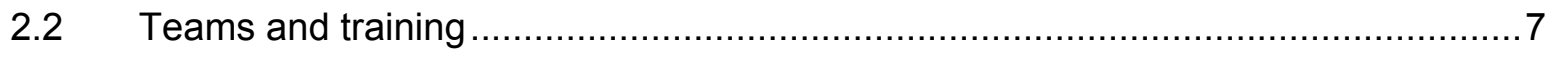

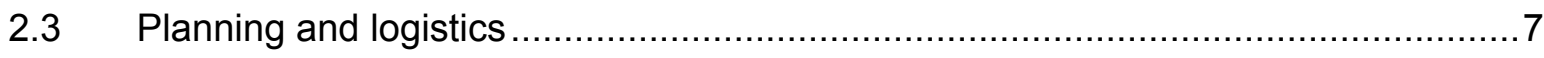

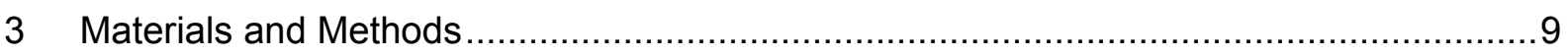

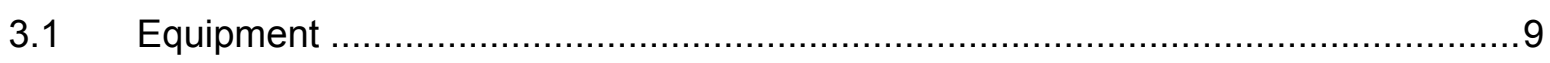

3.2 The absorbed dose measurement method ................................................... 10

3.3 The technical protocol and measurement methods ............................................. 11

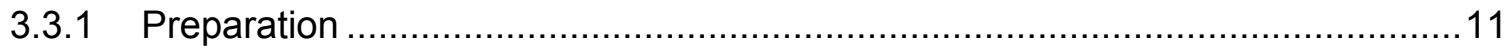

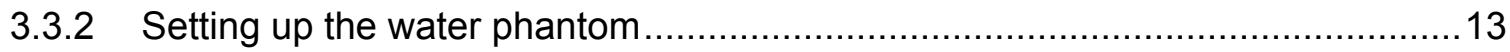

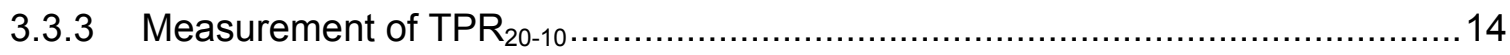

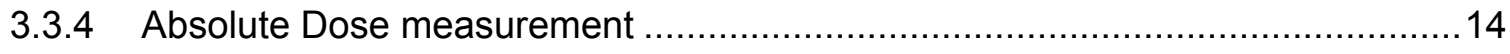

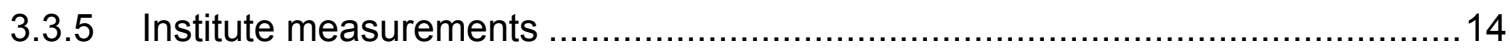

3.3.6 Enclosing the Audit measurements .......................................................... 15

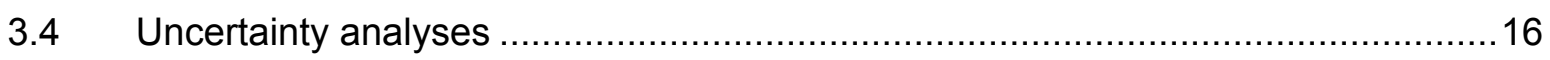

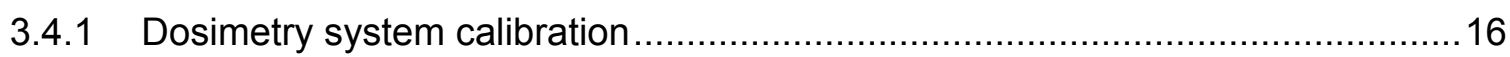

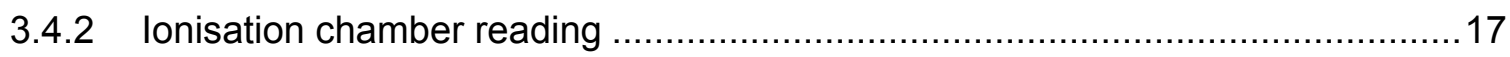

3.4.3 Correction factors for influence parameters .................................................17 


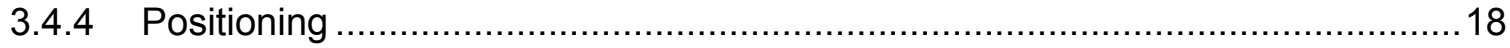

3.4.5 Beam quality correction factor and beam quality index ............................... 19

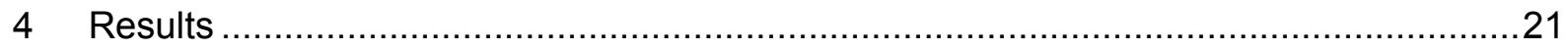

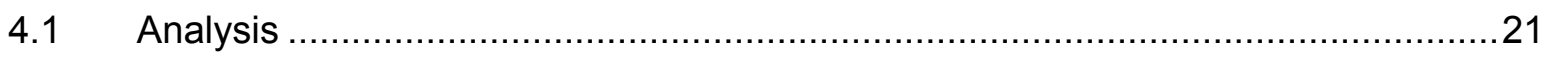

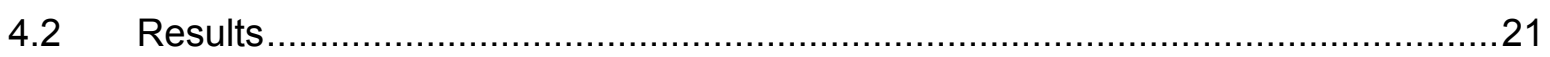

4.2.1 Audit TPR 20,10 vs. Institute $\mathrm{TPR}_{20,10 \ldots \ldots \ldots \ldots \ldots \ldots \ldots \ldots \ldots \ldots \ldots \ldots \ldots \ldots \ldots \ldots \ldots \ldots \ldots \ldots \ldots \ldots \ldots \ldots \ldots \ldots \ldots \ldots \ldots \ldots \ldots \ldots \ldots \ldots \ldots \ldots \ldots \ldots \ldots \ldots} 22$

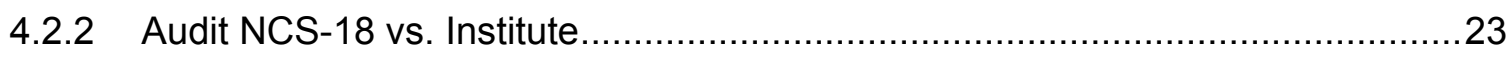

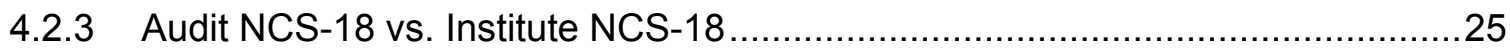

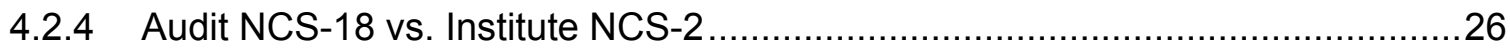

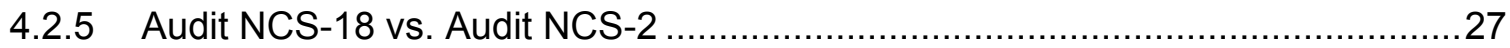

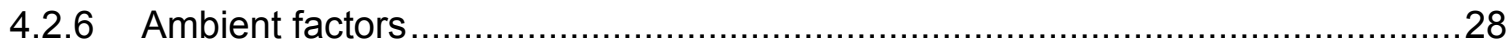

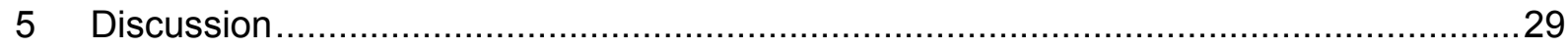

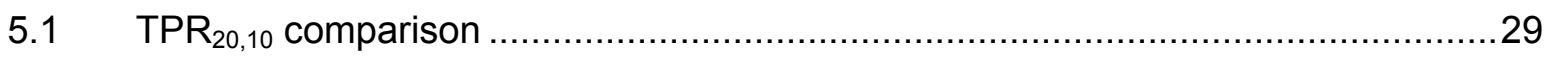

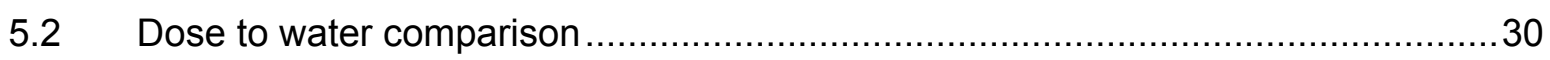

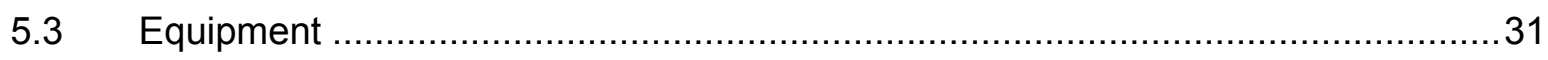

6 Conclusions

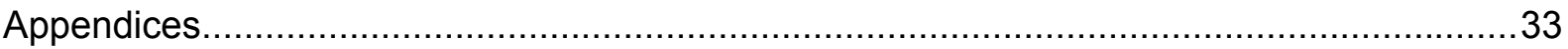

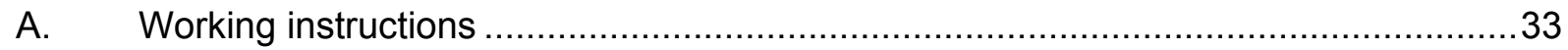

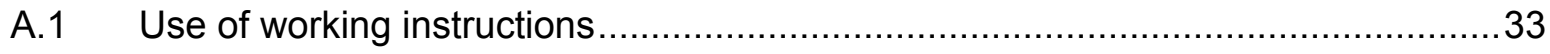

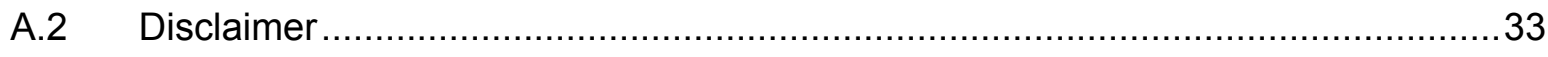

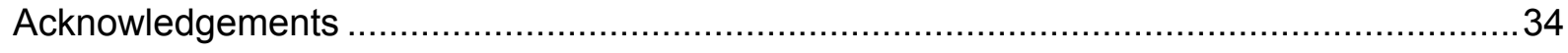

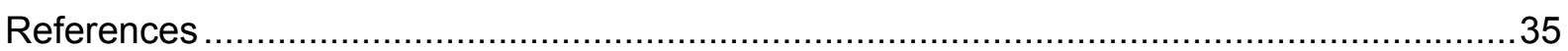




\section{Abbreviations}

AMC Academisch Medisch Centrum, Amsterdam

BHPA Belgian Hospital Physicists Association

CoP $\quad$ Code of Practice

CZE Catharina Ziekenhuis, Eindhoven

EORTC European Organisation for Research and Treatment of Cancer

FANC Federal Agency for Nuclear Control, Belgium

MCA Medisch Centrum Alkmaar, Alkmaar

NCS Nederlandse Commissie voor Stralingsdosimetrie (Netherlands Commission on Radiation Dosimetry)

NCS-18 Nederlandse Commissie voor Stralingsdosimetrie: Code of Practice for the Absorbed Dose Determination in High Energy Photon and Electron Beams, NCS Report 18, January 2008

NCS-2 Nederlandse Commissie voor Stralingsdosimetrie: Code of Practice for the Dosimetry of High energy Photon Beams, NCS Report 2, December 1986

NCS-5 Nederlandse Commissie voor Stralingsdosimetrie: Code of Practice for the Dosimetry of High energy Electron Beams, NCS Report 5, December 1989

NVKFM Dutch Association for Medical Physics Engineers

PMMA Polymethylmethacrylate

QA Quality Assurance 
SCK- $\quad$ Studiecentrum voor Kernenergie - Laboratorium voor Nucleaire Kalibraties, Gent LNK

VSL VSL B.V., Dutch Metrology Institute, Delft

VUmc VU Medisch Centrum, Amsterdam

ZRTI Zeeuws Radiotherapeutisch Instituut, Vlissingen 


\section{List of symbols}

$D_{w, Q} \quad$ Absorbed dose to water in the beam quality $Q$.

$k h \quad$ Factor to correct for the response of an ionisation chamber for the effect of humidity if the calibration coefficient of the chamber is referred to dry air.

kQ,Qo Beam quality correction factor which corrects for the difference in the absorbed dose to water calibration coefficient of a reference beam quality $Q_{0}$ to that of a radiation beam of quality $Q$.

kQ Beam quality correction factor which corrects for the difference in the absorbed dose to water calibration coefficient of a ${ }^{60} \mathrm{Co}$ beam to that of a radiation beam of

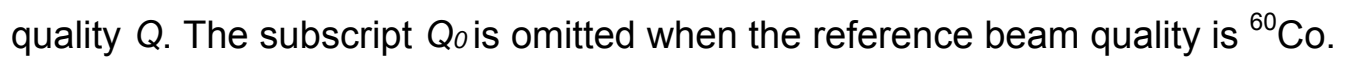

kpol Factor to correct for polarity effects affecting the response of the ionisation chamber.

ks Factor to correct for the response of an ionisation chamber for the lack of complete charge collection.

KTP Factor to correct for the response of an ionisation chamber for deviations of temperature and pressure from reference conditions.

Mcorr, $Q \quad$ The electrometer reading corrected for any difference between the ambient air conditions affecting the ionisation chamber at the time of measurement and the standard ambient air conditions for which the calibration coefficient applied (air temperature, pressure and humidity), for ion recombination and for polarity effects.

MQ The uncorrected reading of the instrument.

$N_{D, w} \quad$ The absorbed dose to water calibration coefficient for an ionisation chamber (or for the dosimeter assembly: ionisation chamber and electrometer) at the reference beam quality ${ }^{60} \mathrm{Co}$. 
$N_{D, w, Q} \quad$ The absorbed dose to water calibration coefficient for an ionisation chamber (or for the dosimeter assembly: ionisation chamber and electrometer) at the beam quality Q.

$N_{k} \quad$ Air kerma calibration coefficient in ${ }^{60} \mathrm{Co}$, given by the standard dosimetry laboratory, which converts the ionization chamber reading to air kerma for the calibration quality and geometry for standard ambient air conditions.

Q Symbol to indicate the quality of a radiation beam.

Qo Symbol to indicate the reference beam quality used for calibration of an ionisation chamber (usually in a Standards Laboratory).

rdg Reading of a dosimeter in arbitrary units.

$r_{\text {cyl }} \quad$ Cavity radius of a cylindrical ionisation chamber.

sd Standard deviation.

SCD Source-chamber distance.

SDD Source-detector distance.

SSD Source-surface distance.

TPR20,10 Tissue-phantom ratio in water at depths of 20 and $10 \mathrm{~cm}$, for a field size of $10 \mathrm{~cm}$ $\times 10 \mathrm{~cm}$ and a SCD of $100 \mathrm{~cm}$, used as the beam quality index for high energy photon radiation

$u_{i} \quad$ Standard uncertainty of a quantity $i$.

$u_{c} \quad$ Combined standard uncertainty of a quantity.

$U \quad$ Polarising voltage applied to the ionisation chamber. 


\section{Introduction}

\subsection{Introduction NCS-18}

In 2008 the Netherlands Commission on Radiation Dosimetry (NCS) issued a new code of practice for high-energy photon and electron beam therapy based on absorbed dose to water standards in a ${ }^{60} \mathrm{Co}$ y-radiation beam [1]. The new code of practice (NCS-18) replaced the air kerma based codes of practice described in NCS Reports 2 and 5, published in respectively 1986 [2] and 1989 [3]. These codes provided a simple formalism to determine the quantity absorbed dose to water under reference conditions using $N_{k}$ calibration coefficients and single conversion factors to convert the reading of an ionisation chamber to absorbed dose to water as a function of radiation quality. The old codes of practice were kept brief and relatively simple and the underlying physics, numerical data for correction and conversion factors were given in appendices. This approach was maintained in NCS-18, where a single beam quality correction factor is introduced taking into account all effects dependent of the radiation beam quality. This beam quality correction factor is defined as the ratio of absorbed dose to water calibration coefficients in the clinical beam quality $Q$ and the reference beam quality $Q_{0}$ :

$$
k_{Q, Q_{0}}=\frac{N_{D, w, Q}}{N_{D, w, Q_{0}}}
$$

Where:

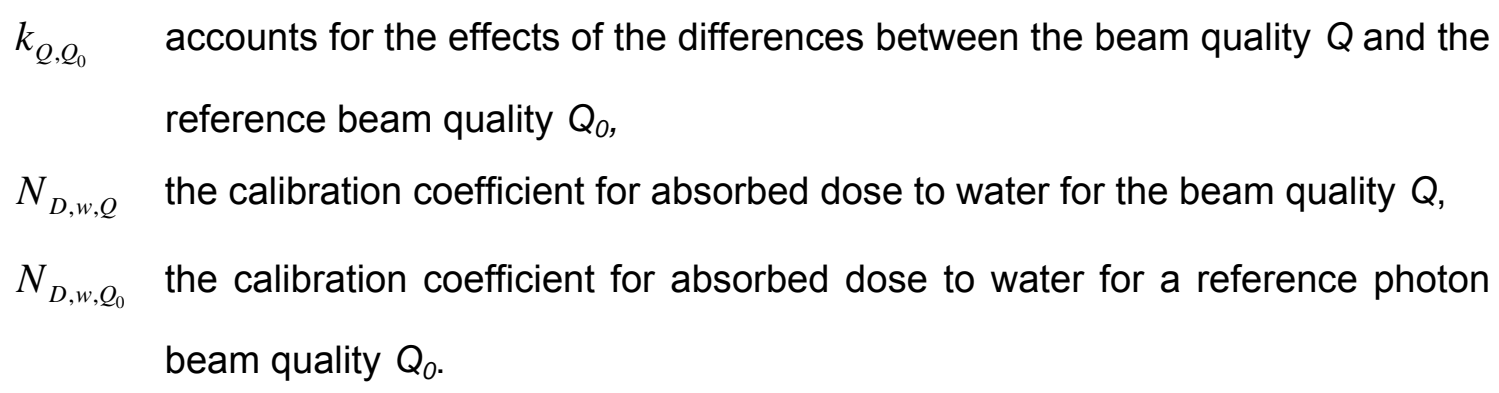

The formalism to determine the absorbed dose to water at the reference depth in water in a beam of quality $Q$ is given by:

$$
D_{w, Q}=M_{c o r r, Q} \cdot N_{D, w, Q_{0}} \cdot k_{Q, Q_{0}}
$$

where: 
$M_{c o r r, Q}$ reading of the electrometer corrected to ambient reference conditions and for the effects of recombination, polarity and the influence of the electrometer,

$N_{D, w, Q_{0}}$ the calibration coefficient for absorbed dose to water for a reference photon beam quality $Q_{0}$,

$k_{Q, Q_{0}}$ accounts for the effects of the differences between the beam quality $Q$ and the reference beam quality $Q_{0}$.

The reference beam quality $Q_{0}$, used for the calibration of ionisation chambers, is ${ }^{60} \mathrm{Co} \mathrm{Y}$ radiation, in which case the symbol $Q_{0}$ is omitted in equation (2). In NCS-18 only graphite walled cylindrical ionization chambers are recommended for reference dosimetry in highenergy photon beams. For photon beams the beam quality correction factors $k_{Q}$ are determined experimentally in selected clinical beams in Belgium and the Netherlands together with measurements of the photon beam quality specifier. The measurements involved the use of a transportable water calorimeter, developed at VSL and four different sets of graphite walled cylindrical ionization chambers (NE 2611A, NE 2571, PTW 30012 and IBA FC65G). Each set consisted of 6 chambers of the same type. The measured $k_{Q}$ values were combined with existing and new experimental data in a model analysis resulting in a simple parametric representation of the data, which is convenient to use in clinical practice. The beam quality for photon beams is specified by the tissue-phantom ratio $T P R_{20,10}$, which is defined as the ratio of the water absorbed doses on the beam axis at depths of $20 \mathrm{~cm}$ and $10 \mathrm{~cm}$ in a water phantom, obtained with a constant source-detector distance of $100 \mathrm{~cm}$ and a $10 \mathrm{~cm} \times 10 \mathrm{~cm}$ field size at the position of the detector. The parameter is a measure of the effective attenuation coefficient describing the approximately exponential decrease of a photon depth-dose curve beyond the depth of maximum dose. Furthermore the parameter is independent of the electron contamination in the incident beam. In the code of practice the $k_{Q}$ data for photon beams are given as function of the beam quality specifier $\mathrm{TPR}_{20,10}$. SCK$\mathrm{LNK}^{1}$ in Belgium and VSL in the Netherlands operate calibration services for absorbed dose to water in ${ }^{60} \mathrm{Co} y$-radiation. All calibrations include the use of a $1 \mathrm{~mm}$ thick waterproofing sheath, made of PMMA. The new code of practice contains appendices giving information on various topics, e.g. on recommended ionization chambers, dosimetry methods, physical and numerical data concerning influence quantities and beam quality correction factors. A special

\footnotetext{
${ }^{1}$ SCK-LNK operates the calibration services in Ghent, which were conducted in the past by LSDG (Laboratory for Standard Dosimetry Ghent).
} 
appendix is devoted to the estimation of uncertainty in dose measurements. The reader is referred to NCS Report 18 [1] for more detailed information on the Code of Practice.

\subsection{History of audits in Belgium and the Netherlands}

\subsubsection{Belgium}

In Belgium there is a long history of dosimetry audits in the radiotherapy departments. In 1987, the Belgian Hospital Physicists Association (BHPA) started a dosimetry program. The goal of this audit program was to verify and to improve the uniformity of dosimetric procedures in radiotherapy. At that time, a number of different dosimetric protocols were in common use. Since then, the BHPA recommends to its members to follow the NCS Dosimetry protocols (NCS Reports 2 [2], 5 [3] and 18 [1]), and to welcome on-site quality control visits. These audits are performed by a voluntary team, on request of the local physicist, in order to check the correct implementation of the advised dosimetry protocols. In the first report done by M.Th. Hoornaert et al [4] in 1993, sixteen of the 31 Belgian radiotherapy centres were visited, i.e. 13 Cobalt units and 12 simulators, 24 high energy Xray beams (from 4 to $25 \mathrm{MV}$ ) produced by 18 linacs, representing about $70 \%$ of all the linacs installed in Belgium at that time.

Since then, a lot of other centres have participated in such audits; the actual quality audit team, still composed of voluntary physicists, has visited 25 centres, since 2005.

In the meantime, the NCS sub-committee Dosimetry Audit and the Federal Agency for Nuclear Control (FANC) have also performed quality audits in several centres in Belgium. The federal dosimetry audit project (named « Beldart ») set up by FANC, is based on Alanine dosimetry and tested all the radiotherapy linacs in Belgium within a period of three years.

\subsubsection{The Netherlands}

The first national dosimetry audit in the Netherlands was part of a research project, "Investigations of differences between dosimetry measurements and treatment planning calculations in the radiotherapy departments in the Netherlands" [5]. The dosimetry intercomparison took place between March and December 1985. During this investigation all 20 radiotherapy departments were visited by one person. Absolute dose measurements with a Baldwin-Farmer type chamber, directly calibrated at the Dutch primary standards dosimetry laboratory, were performed in a water phantom and compared with stated absolute dose measurements. Differences between stated and measured dose values were on the average $0.5 \%$ with a standard deviation of $1.9 \%$, but up to $6 \%$ at maximum. If all institutes apply the 
same set of recommended values for the physical quantities, the maximum difference reduced to $2 \%$, thus showing the importance of using a common dosimetry protocol. Further results are described by Wittkamper et al [5].

In 2005 a regional dosimetry intercomparison was performed between institutes in the southwestern part of the Netherlands [6]. Inspired by this initiative, a similar regional dosimetry intercomparison [7] was carried out in 2007 between the radiotherapy departments in the north western part of the Netherlands (the Netherlands Cancer Institute, AMC, VUmc, and MCA). Absolute dose values were measured by the visiting team with a calibrated chamber and in a water phantom. The dose values were compared with the routine dose values measured by the institute. Differences up to $3 \%$ were seen between the audit measurements and the routine measurements. Looking at the relevant correction and correction factors applied, some factors were modified, resulting in reduced differences.

Beside these multi-institute intercomparisons, dosimetry intercomparisons between the Netherlands Cancer Institute and other institutes (ZRTI, VUmc, MCA) were carried out in the last decade.

\subsection{Need for Audits}

\subsubsection{Establishment of the NCS subcommittee Dosimetry Audits}

During 2006 and 2007 a small dosimetry audit [7], based on the NCS 2 Code of Practice, was initiated by medical physicists in training in the region of North Holland in the Netherlands. During this audit some systematic deviations were found in the absorbed dose calculations and measurements. The deviations found in the audits raised the question if a national audit was required.

Within the NVKFM this idea was adopted and soon an audit committee was formed to organize an absolute dosimetry audit in the Netherlands. This committee contacted the NCS board, which was about to publish a new code of practice for absolute dosimetry. This report was published as NCS-18. The NCS board suggested that this committee could work under the flag of the NCS so Belgian institutes could also be included. In February 2008 the NVKFM committee was officially installed as the NCS subcommittee Dosimetry Audits. The Subcommittee consists of a team of trained dosimetry experts (Medical Physics Engineers and Medical Physicists) from various radiotherapy institutes in Belgium and the Netherlands. 


\subsubsection{Goal of the NCS subcommittee Dosimetry Audits}

The goal of the subcommittee was to design and administer a measurement audit with respect to absolute dosimetry in radiotherapy departments in the Netherlands and Belgium, to verify the agreement in absolute dose measurements.

The subcommittee serves as an independent external quality control for the implementation of the new NCS-18 Code of Practice [1] with respect to high-energy photons.

Furthermore the aim was to devise an audit that would be accepted for use in international clinical trials such as EORTC. 


\section{Audit set-up}

\subsection{Setting up the Audit}

The methods were drafted during a number of meetings and are described in various documents. These documents contained the scope of the audit, the audit protocol and measurement procedures, involving personal and financial resources, time restraints, choices, operation and calibration of equipment as well as practical issues with respect to the measurement procedures, training of auditors and logistics. This resulted in:

- a measurement protocol;

- detailed measurement instructions including a list of equipment (given in appendix A and the NCS website www.radiationdosimetry.org);

- spreadsheets;

- Audit questionnaire.

The measurement protocol describes the metrological choices based on NCS-18 with respect to the practical implementation of the audit.

\subsubsection{Measurement instructions}

The technical implementation of the measurement protocol resulted in a set of detailed measurement instructions which were drafted during separate practical sessions at the Netherlands Cancer Institute by a delegation of subcommittee members. During these sessions test audits were performed using the audit measurement equipment. Earlier proposed methods were tested, validated, discussed and documented. After each session the delegation reported to the subcommittee after which consensus would be reached about the contents of the measurement instructions. It was decided that the measurement instructions should be comprehensive and detailed so that each audit team, consisting of two auditors, would, after adequate training, be able to perform the measurements in an identical way. Therefore the auditors were committed to follow the working instructions accurately. The measurement instructions were set-up in such a way that, when printed, they could be used as worksheets not only requesting measurement values but also information about the radiotherapy department, accelerator type, serial number, radiation beams and other audit information. One of its purposes was to provide for a hard-copy back-up of collected data in order to ensure reliable and traceable results of used methods, materials and data. Therefore they were supplied with a version number. By means of text mark-up and high-lighting, including check-boxes and numbering according to the value entries in the related spreadsheet the auditors were guided through the quality defining measurement steps. 
After completion of the audit visit, the filled-in instruction sheet would be reviewed, scanned and archived. At a later stage it would be used to validate the digital spreadsheets.

\subsubsection{Measurement spreadsheet}

The audit spreadsheet was also created as result of the practical sessions at the Netherlands Cancer Institute. The spreadsheet requests measurement data as well as audit information such as radiotherapy department, accelerator type, serial number, radiation beams and other audit information. Prior to the audit visit it was filled in based on the information provided by the radiotherapy department. At the day of the audit this information would be validated and where needed supplemented or edited.

\subsubsection{Audit questionnaire}

A questionnaire was drafted and distributed prior to the start of the audits. This spreadsheet was send to all radiotherapy departments in Belgium and the Netherlands being potential audit participants. The returned questionnaire provided the subcommittee with general information regarding the departments official name, (satellite) locations, local contact person and responsible medical physicist as well as technical details about the accelerator, available beam qualities, reference conditions, reference protocols and calibrated dosimetry equipment.

\subsection{Teams and training}

Audit teams consisted of two trained auditors being subcommittee members who were considered competent to act as an auditor as a result of their knowledge and experience in the field of dosimetry and QA. In order to ensure reliable and repeatable audit results, training sessions were organized at CZE and the Netherlands Cancer Institute. The aim of the training sessions was to familiarize the auditors with the audit equipment and the practical implementation of the measurement methods. Additionally, during the training sessions clearness of the measurement instructions was tested.

\subsection{Planning and logistics}

Due to the sharing of workload between the committee members, location of the radiotherapy departments and domestic location of the auditors, the audit planning was setup per geographical region (north, Holland, mid, west and south), see Figure 1. 
It was decided that, in principle, members of the subcommittee were not allowed to take part in an audit at their own radiotherapy department, neither as an auditor, nor as a member of the local staff. In several occasions, though not involved in the actual audit, subcommittee members were present during the audit in their own institute.

The audit teams were composed in a relay (estafette) manner where during each audit one auditor would have contributed to the previous audit and the second auditor would be new and contributing to the next audit. This resulted in a logistical benefit where the audit equipment could be transferred to the next location by the second auditor. In some instances, typically after finishing a region, the audit equipment was transported to VSL where it was re-calibrated.

In total 26 audits were performed by all subcommittee members.

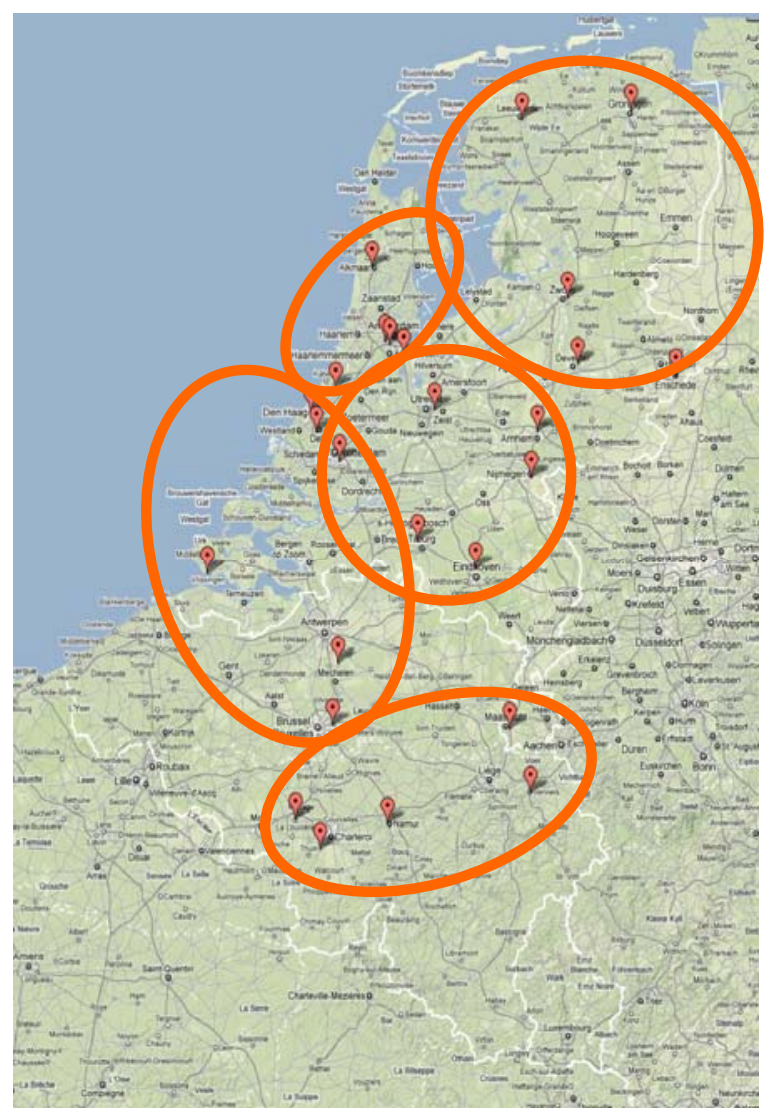

Figure 1: Geographical view of location of regions 


\section{Materials and Methods}

\subsection{Equipment}

For the measurement of the megavoltage photon beam doses the audit team used a Unidos $^{\text {webline }}$ dosemeter (TN10023, PTW-Freiburg) in combination with a cylindrical graphitewalled Farmer-type ionisation chamber (TN30012, PTW-Freiburg). This combination was regularly calibrated at VSL in a ${ }^{60} \mathrm{Co} y$-ray beam. The ionisation chamber was placed inside a custom made waterproof PMMA sleeve with a wall thickness of $1 \mathrm{~mm}$. This combination was mounted in a MP1-M water phantom tank (T41025, PTW-Freiburg) with inner dimensions of $30 \times 30 \times 35 \mathrm{~cm}^{3}$. A silicon tube with diameter of $15 \mathrm{~mm}$ was impermeably connected to the waterproof sleeve in order to keep the ionisation chamber cable dry when submerged.

The phantom tank was equipped with a precision vertical translation stage. The reading of the translation stage was verified at VSL.
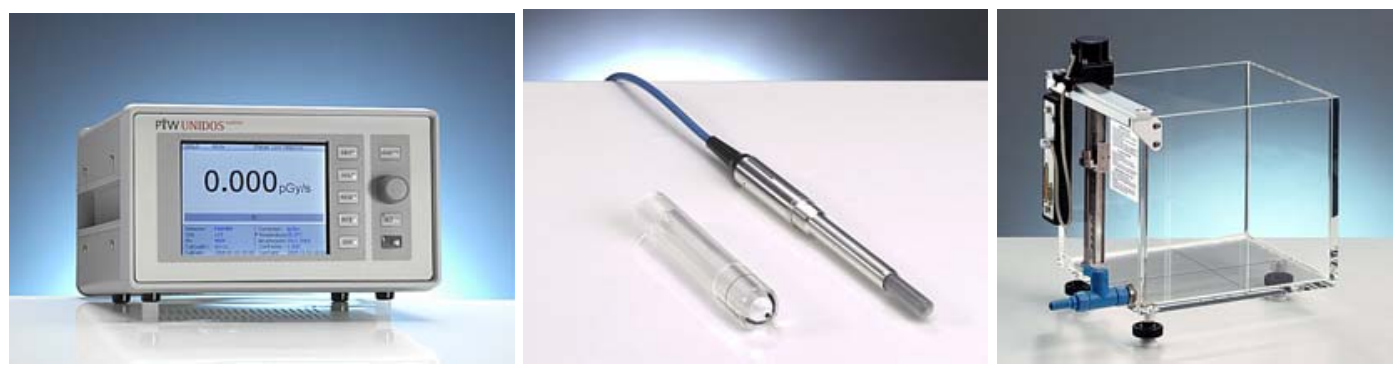

Figure 2: Electrometer (left), farmer type chamber (center) and water phantom (right)

A custom made polystyrene bridge was used to mount the waterproof sleeve on the translation stage's vertical carriage. The purpose of the bridge was to place the chamber in the centre of the phantom and to minimize beam scatter as a result of the beam hitting metal parts of the translation stage.

A desk ruler, of which the reading was verified at VSL, was used to measure the treatment table's displacement in vertical orientation in relation to the laser.

A dedicated barometer (DPI 142, Drück), thermometer (450, Doric) and thermo-hygrometer (Hygroskop GT, Rotronic) were used to determine atmospheric pressure, water temperature, ambient temperature and relative humidity which were used to determine the correction factors applied to the ionisation chamber readings. The thermometer consisted of a handheld unit in combination with a separate thermistor sensor which was placed in the water phantom using a waterproof metal sheet. Additionally the ambient temperature was measured.

All electronic devices were regularly calibrated at VSL and therefore traceable to (inter)nationally accepted measurement standards. 


\subsection{The absorbed dose measurement method}

The audit measurement consisted of the measurement of the absorbed dose to water, $D_{\mathrm{w}, \mathrm{Q}}$, in the users beam $Q$ per monitor unit, $M U$, in the absence of the chamber at the reference depth $z_{\text {ref }}=10 \mathrm{~cm}$, according to:

$$
D_{M U}=\frac{D_{w, Q}}{M U}
$$

Here, $D_{M U}$ is the absorbed dose per monitor unit. The typical number of $M U$ was usually 200 MU, corresponding to 200 cGy under the department's reference conditions. The absorbed dose to water, $D_{\mathrm{w}, \mathrm{Q}}$, is determined according to NCS-18 as described in formula (2).

The electrometer reading, corrected for influence quantities, $M_{\text {corr, } Q}$ is given by:

$$
M_{\text {corr }, Q}=M_{Q} \cdot k_{T P} \cdot k_{h} \cdot k_{p o l} \cdot k_{s}
$$

Where:

$M_{Q} \quad$ is the uncorrected electrometer reading,

$k_{T P} \quad$ is the correction to reference temperature and pressure, $T_{\text {ref }}$ and $P_{\text {ref }}$ at which the calibration coefficient $N_{D, \mathrm{w}}$ is valid,

$k_{h} \quad$ is the correction for the humidity in the air cavity at the time of measurement in relation to the humidity at which the calibration coefficient is valid. Since the $N_{D, w}$ is valid for a relative humidity between $20 \%$ and $80 \%$ and the audit measurements were done at the same humidity range, $k_{h}$ was taken to be unity,

$k_{\text {pol }} \quad$ is the correction on the chamber reading due to the applied polarity. Since the chamber was calibrated at the same polarity at which it was used during the audit, $+400 \mathrm{~V}$, this correction was taken to be unity,

$k_{s} \quad$ is the correction for incomplete charge collection in the ionisation chamber due to recombination. This correction was measured using the recommended method by NCS-18 and described by Weinhous and Meli [8].

$N_{D, \mathrm{w}}$ is the absorbed dose to water calibration coefficient in the ${ }^{60} \mathrm{Co}$ reference beam quality at reference ambient conditions of $T_{\text {ref }}=293.15 \mathrm{~K}$ and $P_{\text {ref }}=101.325 \mathrm{kPa}$ and $50 \%$ relative 
humidity. $k_{Q}$ is the beam quality correction factor that depends on the chamber type and radiation beam quality index, $T P R_{20,10}$. It is determined according to NCS-18:

$$
k_{Q}=0.80+0.20 \frac{1+e^{C\left(0.57-X_{0}\right)}}{1+e^{C\left(T P R_{20,10}-X_{0}\right)}}
$$

For the recommended graphite-walled cylindrical ionisation chambers of the type used by the audit team, the PTW TN30012, the parameter $C=11.67$ and $X_{0}=0.9198$.

The beam quality index, $T P R_{20,10}$, is determined according to the description in NCS-18 where the ratio is taken between the absorbed dose measurement at a depth of $20 \mathrm{~cm}$ and $10 \mathrm{~cm}$ with a constant source chamber distance, $S C D$, of $100 \mathrm{~cm}$, effectively resulting in the ratio of charge measurements, corrected for temperature and pressure:

$$
T P R_{20,10}=\frac{M_{Q, 20 \mathrm{~cm}} k_{P T, 20 \mathrm{~cm}}}{M_{Q, 10 \mathrm{~cm}} k_{P T, 10 \mathrm{~cm}}}
$$

The $T P R_{20,10}$ result, $\triangle T P R_{20,10}$, consisted of a comparison of the $T P R_{20,10}$ measured by the NCS, $T P R_{20,10, N C S}$ and measured or supplied by the radiotherapy department, $T P R_{20,10, i}$ :

$$
\Delta T P R_{20,10}=\frac{T P R_{20,10, i}-T P R_{20,10, N C S}}{T P R_{20,10, N C S}} \cdot 100 \%
$$

The audit result, $\Delta D_{M U}$, consisted of a comparison between $D_{M U}$ measured by the NCS, $D_{M U, N C S}$, and $D_{M U}$ measured by the radiotherapy department, $D_{M U, \mathrm{i}}$. For each measurement $200 \mathrm{MU}$ were given, representing 200 cGy under reference conditions.

$$
\Delta D_{M U}=\frac{D_{M U, i}-D_{M U, N C S}}{D_{M U, N C S}} \cdot 100 \%
$$

The comparison was based on the substitution method where the department's measurements were preceded and followed by the NCS measurements.

\subsection{The technical protocol and measurement methods}

\subsubsection{Preparation}

Prior to the audit visit, the radiotherapy department received a letter containing the following information:

- the date of the audit visit, as agreed earlier;

- the members of the audit team and the subcommittee contact person; 
- how to prepare for the audit visit (e.g. preparation of 40 litres of water, to send information regarding $P D D$-values, $T P R_{20,10}, k_{\mathrm{Q}, \mathrm{Q} 0}$ to the subcommittee, etc.);

- a short description of the audit protocol.

The radiotherapy department was requested to prepare 40 litres of clean, preferably demineralised, water and leave this in the treatment room for at least 24 hours to acclimatize. This water was used in the audit phantom.

On the day of the audit, the audit team typically arrived around noon and left before the evening. Upon arrival the equipment was unpacked in the treatment room, distributed to its location of operation and, if not battery operated, switched on. This allowed for acclimatization of the equipment. During lunch, the program of the day was discussed with the local staff.

Subsequently the team prepared the audit by checking the availability of the audit spreadsheet and measurement instructions and asked the local staff to position the accelerator gantry position at $0^{\circ}$ (vertical beam) and the collimator to $0^{\circ}$ or $90^{\circ}$, depending on the settings during the department's reference dosimetry. The field size was adjusted by the local staff to $10 \times 10 \mathrm{~cm}^{2}$ at the isocenter.

The position of the light ruler and the laser in relation to the isocenter was checked according to the local protocol. Any deviations would be reported but not adjusted. The local staff was informed that the audit measurement was going to be performed using the local Source Surface Distance (SSD) and Source Chamber Distance (SCD), according to the current geometrical settings in order to avoid ambiguous audit results, not exclusively caused by dosimetrical aspects. 


\subsubsection{Setting up the water phantom}

The NCS water phantom was placed on top of the treatment table, filled with water and levelled. The water surface was first set to an $S S D$ of $100 \mathrm{~cm}$ (isocenter) by means of the light ruler and lasers. The desk ruler (see Figure 1), placed vertically on the side of the phantom, was used to mark the position of the horizontal laser. Subsequently the treatment table was moved up by $20.0 \mathrm{~cm}$ $(S S D=80 \mathrm{~cm})$ using the displacement of the desk ruler in relation to the laser. The displacement was verified by reading the table's translation. Any deviation would be reported. By means of this method, the vertical positioning of the phantom could be

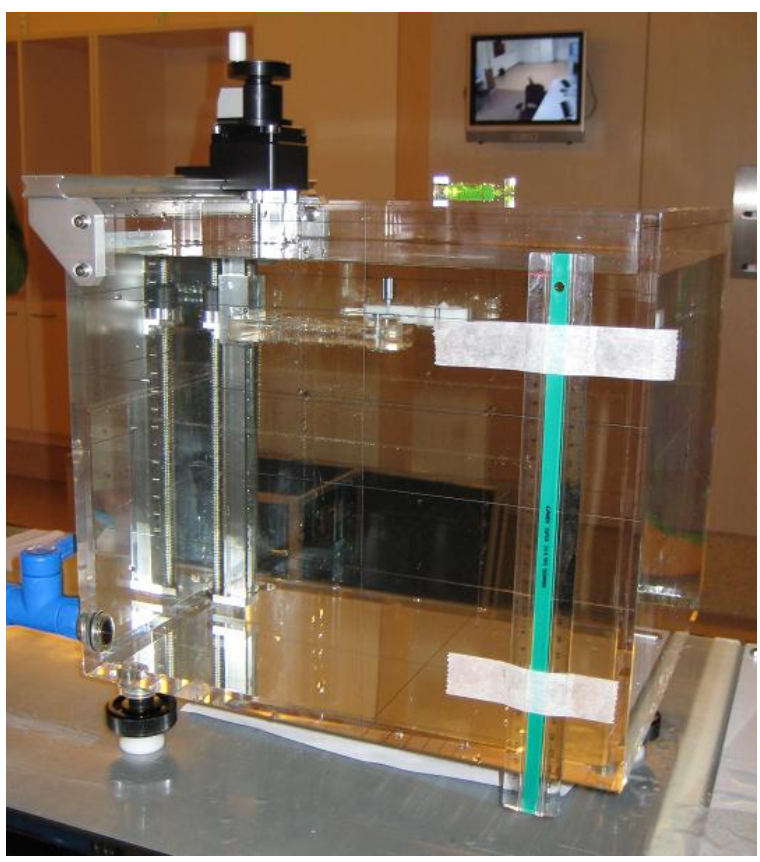

Figure 3: Water phantom with desk ruler done well within $0.5 \mathrm{~mm}$.

At this point the ionisation chamber was placed inside its waterproof sleeve. The silicon tube was connected to the sleeve, keeping the cable and chamber dry when submerged. The assembly was placed on the bridge support, connected to the carriage of the vertical translation stage and the chamber, placed in the centre of the beam, was moved to a depth of $20.0 \mathrm{~cm}(S C D$ of $100 \mathrm{~cm})$.

As a result of the introduction of the waterproof sleeve in combination with the silicon tube, the water surface significantly changed during submersion of the ionisation chamber assembly. Additionally, as a result of the mechanical stress on the waterproof sleeve caused by the relatively thick silicon tube, a potential error was made with respect to the depth position of the chamber. These effects have been taken into account and are described in 3.4 .4

After this, the temperature sensor, placed in a waterproof metal sleeve, was positioned inside the water phantom, just outside the radiation beam. The chamber was electrically connected to the electrometer via a $20 \mathrm{~m}$ extension cable and a bias voltage of $-400 \mathrm{~V}$ was applied to its collecting electrode by setting the electrometer bias voltage to $+400 \mathrm{~V}$. After at least 15 minutes of applied bias voltage the chamber was pre-irradiated with $800 \mathrm{MU}$. 
Each dose measurement consisted of at least 5 charge measurements, preceded and succeeded by measurements of the room temperature, water temperature, ambient pressure and relative humidity. All the measurement values were entered in the spreadsheet and on the instruction worksheet. One leakage measurement was performed just before the first measurement of the $20 \mathrm{~cm}$ dose point of the $T P R_{20,10}$ (after the first pre-irradiation). Leakage currents smaller than $10 \mathrm{fA}$ were considered negligible. Currents larger than $10 \mathrm{fA}$ needed to be reduced before continuing the measurements.

\subsubsection{Measurement of $T P R_{20-10}$}

After pre-irradiation of the ionisation chamber, the $20 \mathrm{~cm}$ dose point for the $T P R_{20,10}$ was measured. Subsequently, the ionisation chamber was moved up by $10.0 \mathrm{~cm}$ and the treatment table moved down by $10.0 \mathrm{~cm}$ to an $S S D$ of $90 \mathrm{~cm}$, respectively using the phantom's vertical translation stage and the desk ruler on the side of the phantom based on the position of the horizontal laser. In the new position, the ionisation chamber's SCD was effectively unchanged and the water depth reduced from $20 \mathrm{~cm}$ to $10 \mathrm{~cm}$. At this position the $10 \mathrm{~cm}$ dose point was measured and the $T P R_{20,10}$ calculated and verified with the value provided by the department in order to determine the $k_{\mathrm{Q}, \mathrm{Q} 0}$ value of the ionisation chamber.

\subsubsection{Absolute Dose measurement}

In case the radiotherapy department defined their dose reference point at an SSD of $90 \mathrm{~cm}$ and a depth of $10 \mathrm{~cm}$, the last $T P R_{20,10}$ dose measurement would also serve as the NCS dose measurement. If the dose reference point was defined at an $S S D$ of $100 \mathrm{~cm}$ and a depth of $d_{\text {max }}$, then the treatment table was moved down by $10.0 \mathrm{~cm}$ before the NCS dose measurement could be performed (SSD of $100 \mathrm{~cm}$ ).

Subsequent to the NCS dose measurement the recombination correction was measured by changing the electrometer bias voltage from $+400 \mathrm{~V}$ to $+133 \mathrm{~V}$ and using the method described by Weinhous and Meli [8].

\subsubsection{Institute measurements}

After the NCS absorbed dose measurement, the radiotherapy department was asked to perform two dosimetry measurements:

1. an absorbed dose measurement following their protocol according to NCS-18, NCS-2 or their in-house method;

2. a routine dose measurement that normally is used for output measurements. 
During these measurements the audit team made notes about the used method and recorded water temperature, atmospheric pressure and relative humidity to exclude dosimetric differences introduced by equipment. Where possible the audit team performed these measurements simultaneously.

\subsubsection{Enclosing the Audit measurements}

After the department's measurements, the dosimetric results, $D_{\mathrm{MU}}$, of the audit team and the radiotherapy department were compared. If deviations were detected (>1.0\%) action was taken immediately. In case of no deviation the audit results were verified and analysed later. The audit was concluded with a final dosimetric measurement of the audit team after setting up the water phantom and measurement equipment again.

Within two weeks after the audit visit, the results were reviewed by an appointed delegation of the subcommittee. Any inconsistencies found in either the audit results or the contents of the measurement instructions were reported to the subcommittee chairman and, if needed, adequate action was taken. The filled-in instructions sheet measurement were scanned, and archived as well as the audit spreadsheet. Preliminary results were sent to the department. 


\subsection{Uncertainty analyses}

Understanding the uncertainty budget is required in order to establish criteria for the determination of 'out of tolerance' results of the audit.

The uncertainty budget, determined according to [9] and [10], is presented in Table 1 and explained in the following paragraphs. For sake of clarity and simplicity, the sensitivity coefficients have not been mentioned in the table because, stated in terms of relative values, these are equal to unity for most contributions.

Table 1 Uncertainty budget for the absorbed dose to water measurement applicable for the audit measurement.

\begin{tabular}{|c|c|c|}
\hline Source of uncertainty & $\begin{array}{c}\text { Relative standard } \\
\text { uncertainty (\%) }\end{array}$ & Par. \\
\hline Dosimetry system calibration & & 3.4 .1 \\
\hline$N_{D w}$, calibration of dosimetry system in ${ }^{60} \mathrm{Co}$ & 0.55 & \\
\hline LT- $N_{D w}$, Long-term stability of $N_{D w}$ & 0.06 & \\
\hline lonisation chamber reading & & 3.4 .2 \\
\hline$M_{\text {res }}$, resolution of dosimetry system $(0.01 \mathrm{nC})$ & 0.01 & \\
\hline M, single measurement series (typical reading) & 0.06 & \\
\hline Correction factors for influence parameters & & 3.4 .3 \\
\hline Temperature measurement for $k_{T}$ at $20^{\circ} \mathrm{C}$ & 0.06 & \\
\hline Pressure measurement for $k_{p}$ at $101.33 \mathrm{kPa}$ & 0.01 & \\
\hline Humidity correction for $k_{R H}=0.998$ at $50 \%$ & 0.03 & \\
\hline Recombination correction, $k_{s}$ & 0.1 & \\
\hline Polarity correction, $k_{\text {pol+ }}$ & 0.12 & \\
\hline Correction factors for positioning & & 3.4 .4 \\
\hline Source detector distance at 900 or $1000 \mathrm{~mm}$ & 0.14 & \\
\hline Depth in water at $100 \mathrm{~mm}$ & 0.31 & \\
\hline Lateral position at the beam centre & 0.10 & \\
\hline Beam quality correction factor & & 3.4 .5 \\
\hline Beam quality correction factor from NCS-18 & 0.40 & \\
\hline Determination of $T P R_{20,10}$ & 0.06 & \\
\hline Combined standard uncertainty & 0.79 & \\
\hline Expanded uncertainty $(\mathrm{k}=2)$ & 1.6 & \\
\hline
\end{tabular}

\subsubsection{Dosimetry system calibration}

The uncertainty contribution of the dosimetry system calibration consists of two components:

1. the calibration coefficient of the dosimetry system;

2. the long-term stability of the dosimetry system calibration coefficient.

The uncertainty of the calibration coefficient of the ionisation chamber and electrometer, calibrated at VSL as a dosimetry system, amounts to $1.1 \%(k=2)$. 
Between November 2009 and August 201115 calibrations were performed. The resulting standard deviation was $0.06 \%$. This value is used as the contribution to the long termstability of the dosimetry system.

\subsection{2 lonisation chamber reading}

The uncertainty contribution of the ionisation chamber reading consists of two components:

1. the resolution of the dosimetry systems display, i.e. $0.01 \mathrm{nC}$;

2. the statistical uncertainty on the readings.

The resolution of the electrometer is $0.01 \mathrm{nC}$ and as a uncertainty contribution is considered to be rectangular, the corresponding standard uncertainty is $0.003 \mathrm{nC}(k=1)$.

The standard deviation of the mean of the electrometer reading was found to be approximately $0.02 \mathrm{nC}(k=2)$ on average and is used as a uncertainty contribution.

\subsubsection{Correction factors for influence parameters}

The uncertainty contribution of the correction factors for influence parameters consists of five components:

1. the correction for temperature of the air in the ionisation chamber cavity;

2. the correction for pressure of the air in the ionisation chamber cavity;

3. the correction for relative humidity of the air in the ionisation chamber cavity;

4. the correction for ion recombination in the ionisation chamber;

5. the correction for polarity effects of the ionisation chamber at positive applied voltage, resulting in a negative voltage at the collecting electrode with respect to the chamber wall.

The temperature of the ionisation chamber was measured with a thermometer present in the water phantom at approximately $50-100 \mathrm{~mm}$ lateral distance from absorbed dose reference point. The uncertainty in the temperature measurement results from the thermometer calibration at $\mathrm{VSL}, 0.15^{\circ} \mathrm{C}(k=2)$, and the potential temperature difference between the thermometer and ionization chamber air cavity. The latter is difficult to determine but estimated to be better than $0.3^{\circ} \mathrm{C}(k=2)$.

The pressure of the air inside the air cavity is determined by means of digital barometer located in the same room as the water phantom, approximately at the same height as the ionization chamber. The uncertainty in the pressure measurement results from the barometer calibration at VSL, $0.05 \mathrm{kPa}$, and the potential pressure difference between barometer and ionization chamber air cavity. The latter is difficult to determine but estimated to be better than $0.2 \mathrm{kPa}(k=2)$. 
The relative humidity of the air inside the air cavity is determined by means of a hygrometer located in the same room as the water phantom. The uncertainty in the humidity measurement is a result from the hygrometer calibration at VSL, $1.5 \%_{\mathrm{RH}}$, and the potential humidity difference between hygrometer and ionization chamber air cavity. However, the humidity correction is a constant value of $k_{h}=0.998$ over a range between 20 and $80 \%{ }_{R H}$ with a maximum deviation of $0.1 \%$ [11]. Because the chamber was calibrated at a relative humidity around $50 \%$ and used at a relative humidity in the range between $20-80 \%$, no correction was applied but an uncertainty contribution was added of $0.1 \%(k=3)$.

The correction for recombination is determined according to the method described in section 3.3.4 and is the result of ionization chamber measurements at two bias voltages, assuming that all other influence parameters are constant and that the uncertainty on the literature provided by [8] is negligible. This uncertainty results solely from the statistical uncertainties of the two charge measurements with the dosimetry system, $0.1 \%(k=1)$.

Polarity is not measured since the audit chamber is calibrated at the same polarity as it is used during the audit measurements, therefore it is assumed to be negligible within the relative uncertainty of $0.12 \%(k=1)$.

The relative uncertainty components transfer directly to the relative uncertainty of the audit dose measurement, i.e. the sensitivity coefficient is unity.

\subsubsection{Positioning}

The audit phantom water surface was positioned according to the local reference position and method (i.e. light ruler and / or lasers).

The uncertainty contribution for the positioning of the ionisation chamber is a result of the potential difference in positioning of the audit equipment to the local equipment and consists of three components:

1. the position of the water surface at a distance of $900 \mathrm{~mm}$ or $1000 \mathrm{~mm}$ from the accelerator target;

2. the position of the ionization chamber at a depth of $100 \mathrm{~mm}$;

3. the position of the ionization chamber in relation to the beam axis.

The uncertainty contribution with respect to the positioning of the ionization chamber at an SDD of $1000 \mathrm{~mm}$ or $1100 \mathrm{~mm}$, respectively SSD of $900 \mathrm{~mm}$ and $1000 \mathrm{~mm}$, related to the local measurement set-up was estimated to be better than $1 \mathrm{~mm}(k=2)$. It was discovered that due to mechanical stress on the waterproof sleeve, the chamber could slightly move up or down and an extra uncertainty contribution of $0.5 \mathrm{~mm}(k=2)$ was introduced, making the 
overall uncertainty on the positioning of the ionization chamber $1.1 \mathrm{~mm}(k=2)$. Because of the square-law with respect to the dose-SDD relation, a sensitivity coefficient of 2 applies and the relative standard uncertainty amounts to $0.11 \%(k=1)$.

The uncertainty with respect to the positioning of the ionization chamber at a depth of $100 \mathrm{~mm}$ is estimated to be $1 \mathrm{~mm}(k=2)$. Due to the mechanical stress, mentioned above, also an uncertainty contribution of $0.5 \mathrm{~mm}(k=2)$ was introduced. Additionally, it was discovered that, as a result of the volume of the water proof sleeve and the silicone tube the water surface would increase with increasing measurement depth. A correction for this effect was determined at VSL and an additional uncertainty to this correction of $0.1 \mathrm{~mm}(k=2)$ was introduced.

Finally an uncertainty is applied for the potential difference in the dose measurement as a result of positioning the chamber off-axis. This was estimated to be better than $0.2 \%(k=2)$ in dose.

Note that knowledge about the exact position of the accelerator target is not needed, but that the uncertainty arises due to the difference in the position of the audit chamber and the institute's chamber.

\subsubsection{Beam quality correction factor and beam quality index}

The uncertainty contribution for the beam quality correction consists of two components:

1. the uncertainty on the beam quality correction factor, $k_{Q, Q O}$ as stated in NCS-18;

2. the uncertainty in determination of $T P R_{20,10}$, used to calculate the $k_{Q, Q 0}$ from the data provided by NCS-18.

The uncertainty of the beam quality correction factor $k_{Q, Q O}$ according to NCS-18 is $0.4 \%$ $(k=2)$. Additionally the uncertainty on determination of the $T P R_{20,10}$ budget has been determined to be $0.5 \%(\mathrm{k}=2)$, resulting in a contribution to the calculation of $\mathrm{k}_{\mathrm{Q}, \mathrm{Q} 0}$ of $0.06 \%$ $(k=2)$. The uncertainty budget of $T P R_{20,10}$ is presented in Table 2. 
Table 2 Uncertainty budget for determination of the beam quality parameter, $T P R_{20,10}$ applicable for the audit measurement

\begin{tabular}{|c|c|}
\hline Source of uncertainty & $\begin{array}{c}\text { Relative standard } \\
\text { uncertainty (\%) }\end{array}$ \\
\hline \multicolumn{2}{|l|}{ Ionisation chamber measurements } \\
\hline Ionization chamber reading at $20 \mathrm{~cm}$ depth & 0.05 \\
\hline Ionization chamber reading at $10 \mathrm{~cm}$ depth & 0.03 \\
\hline \multicolumn{2}{|l|}{ Correction for variation in environmental conditions } \\
\hline Temperature measurement for $k_{T}$ at $20^{\circ} \mathrm{C}$ & 0.05 \\
\hline Pressure measurement for $k_{p}$ at $101.33 \mathrm{kPa}$ & 0.01 \\
\hline Relative humidity correction for $k_{R H}$ at $50 \%$ & 0.00 \\
\hline Recombination correction, $k_{s}$ & 0.07 \\
\hline \multicolumn{2}{|l|}{ Correction for variation in positioning } \\
\hline Source detector distance at $1000 \mathrm{~mm}$ & 0.13 \\
\hline Depth in water at $100 \mathrm{~mm}$ & 0.12 \\
\hline Lateral position at the beam centre & 0.14 \\
\hline \multicolumn{2}{|l|}{ Correction for variation of $k_{Q, Q O}$ with depth } \\
\hline Energy dependence of $k_{Q, Q O}$ & 0.05 \\
\hline Combined standard uncertainty & 0.25 \\
\hline Expanded uncertainty $(\mathrm{k}=2)$ & 0.51 \\
\hline
\end{tabular}




\section{Results}

\subsection{Analysis}

During the audit visit not all departments were able to measure according to the NCS-18. In most cases this was due to the fact that the department did not have the dose to water calibration at its disposal. Therefore the analysis of the audit results is divided in multiple sections.

- Comparison for $T P R_{20,10}$ measurements (\$4.2.1);

- Comparison for dose to water following NCS-18 (audit) versus the protocol used in the department (§4.2.2);

- Comparison for dose to water following only NCS-18, audit and department, where possible (§4.2.3);

- Comparison for dose to water following NCS-18, performed by the audit team, versus NCS-2, performed by the department $(\S 4.2 .4)$;

- Comparison for dose to water following NCS-18 and NCS-2, performed by the audit team;

\subsection{Results}

In total 26 departments have been audited. In each department, at one linac, two or three photon beam qualities were measured, depending on the specific machine's set-up. This results in a total of 58 photon beams. The energies varied from $4 \mathrm{MV}$ to $23 \mathrm{MV}$ and were divided in 3 groups, namely $X_{\text {low }}, X_{\text {med }}$ and $X_{\text {high }}$. Specific energy information is given in Table 3.

Table 3: Energy information

\begin{tabular}{|c|c|c|c|c|}
\hline & \multirow[b]{2}{*}{$\#$} & \multicolumn{2}{|c|}{$T P R_{20,10}$} & \multirow[b]{2}{*}{ Energy } \\
\hline & & from & to & \\
\hline$X_{\text {Iow }}$ & 26 & 0.639 & 0.684 & $4 \mathrm{MV}, 6 \mathrm{MV}$ \\
\hline$X_{\text {med }}$ & 14 & 0.728 & 0.740 & $10 \mathrm{MV}$ \\
\hline$X_{\text {high }}$ & 17 & 0.754 & 0.780 & $15 \mathrm{MV}, 18 \mathrm{MV}, 23 \mathrm{MV}$ \\
\hline Total & 58 & 0.639 & 0.780 & \\
\hline
\end{tabular}


In the following figures the deviations of the institute values are displayed compared to the audit values, using formulas (7) and (8) as given in 3.2. In the following tables the number of measurements, maximum deviation, average and standard deviation (SD) are given.

\subsubsection{Audit $T P R_{20,10}$ vs. Institute $T P R_{20,10}$}

Figure 4 and Figure 5 show the $T P R$ result, $\triangle T P R_{20,10}$, of the stated $T P R_{20,10}$ values from the department to the measured $T P R_{20,10}$ values by the audit team. Results are categorized by $T P R_{20,10}$ range as defined in Table 3.

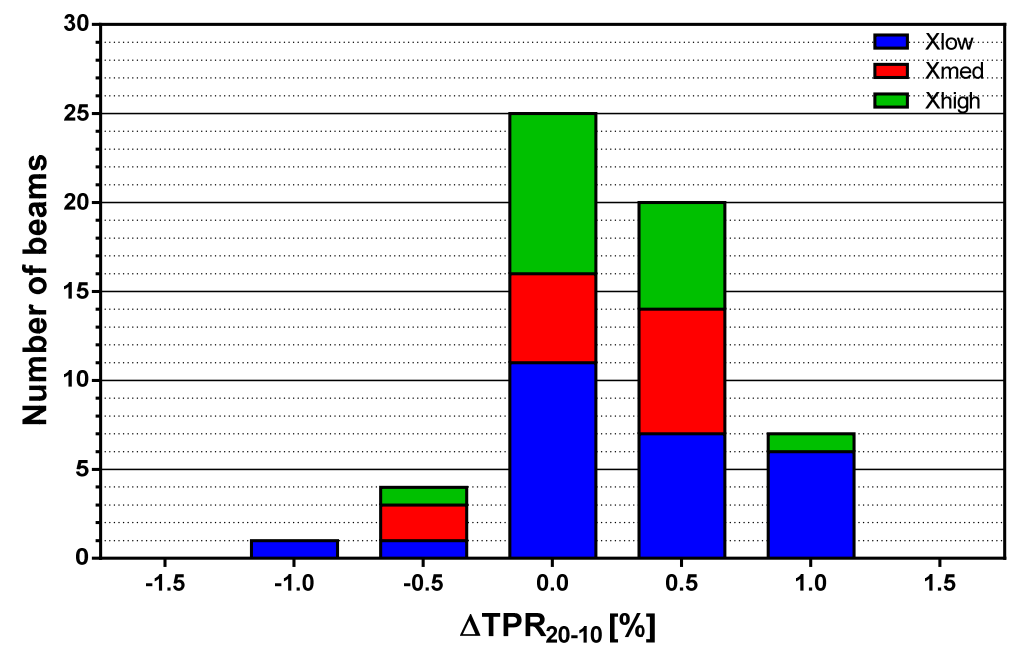

Figure 4: Results for the $T P R_{20,10}$ measurements showing the distribution of the relative difference of $T P R_{20,10}$ measurements performed by the institute and the $T P R_{20,10}$ measurements performed by the audit committee for all 3 energy ranges. 


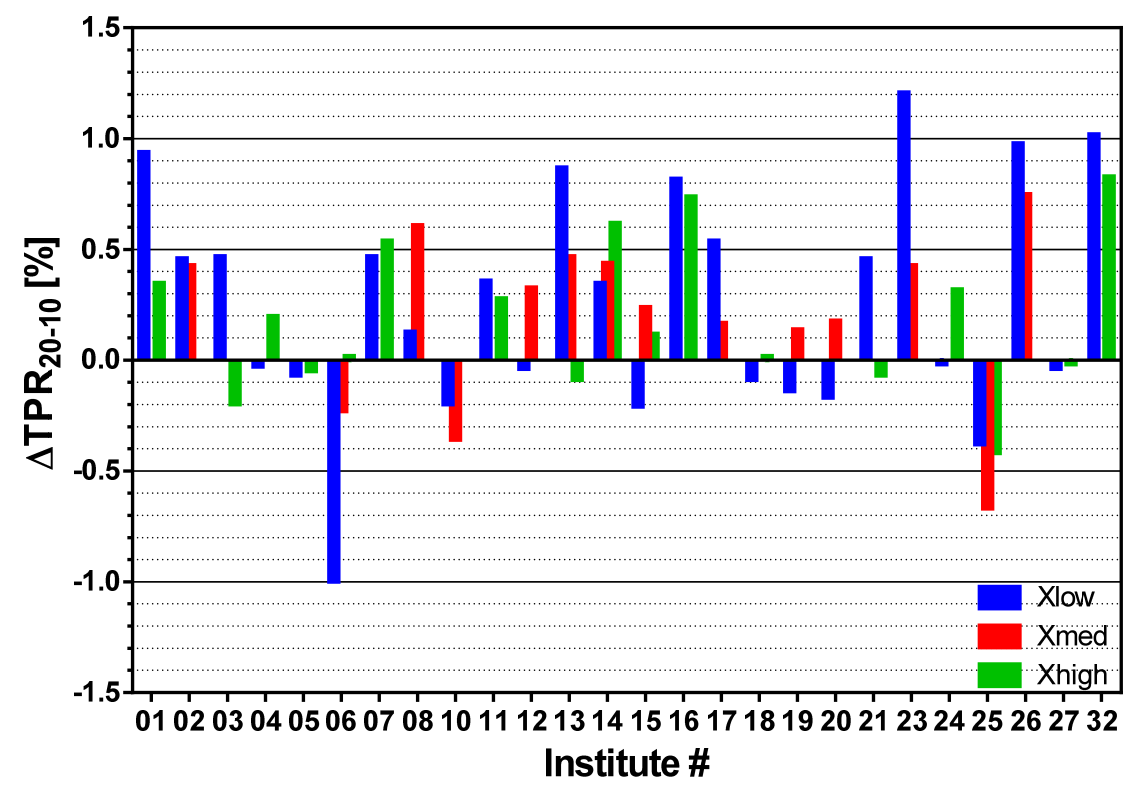

Figure 5: Results for the $T P R_{20,10}$ measurements showing the relative difference of $T P R_{20,10}$ measurements performed by the institute and the $T P R_{20,10}$ measurements performed by the audit committee per institute for all 3 energy ranges.

Table 4 shows the results of the $T P R_{20,10}$ comparison. The maximum deviation from the audit measurements was $1.2 \%$ for $X_{\text {low. }}$. To analyse the impact of this deviation to the absolute dose measurement the difference in $k_{Q}$ factor is calculated. Results of this calculation are shown in Table 5. Due to the small response in $k_{Q}$ factor to errors in $T P R_{20,10}$, no criteria have been set to the deviation for the $T P R_{20,10}$ measurement.

Table 4: Results for the relative difference of $T P R_{20,10}$ measurements performed by the institute and the $T P R_{20,10}$ measurements performed by the Audit committee per institute for all 3 energy ranges.

\begin{tabular}{c|ccc}
\hline & $\mathbf{X}_{\text {low }}$ & $\mathbf{X}_{\text {med }}$ & $\mathbf{X}_{\text {high }}$ \\
\hline \# beams & 26 & 14 & 17 \\
Max & $1.20 \%$ & $0.74 \%$ & $0.82 \%$ \\
Average & $0.26 \%$ & $0.20 \%$ & $0.18 \%$ \\
SD & $0.51 \%$ & $0.38 \%$ & $0.34 \%$ \\
\hline
\end{tabular}

Table 5: Results of the $k_{Q}$ deviations resulting from the deviations measured in $T P R_{20,10}$

\begin{tabular}{c|ccc}
\hline & $\mathbf{X}_{\text {low }}$ & $\mathbf{X}_{\text {med }}$ & $\mathbf{X}_{\text {high }}$ \\
\hline & & & \\
Max & $-0.10 \%$ & $-0.12 \%$ & $-0.22 \%$ \\
Average & $-0.02 \%$ & $-0.03 \%$ & $-0.05 \%$ \\
SD & $0.04 \%$ & $0.06 \%$ & $0.08 \%$ \\
\hline
\end{tabular}

\subsubsection{Audit NCS-18 vs. Institute}

Figure 6 and Figure 7 show the audit result, $\Delta D_{\mathrm{MU}}$, of the measured absorbed dose to water by the institute to the measured absorbed dose to water by the audit team. Results are 
categorized by $T P R_{20,10}$ range as defined in Table 3. In all cases the Audit measurements are performed following NCS-18.

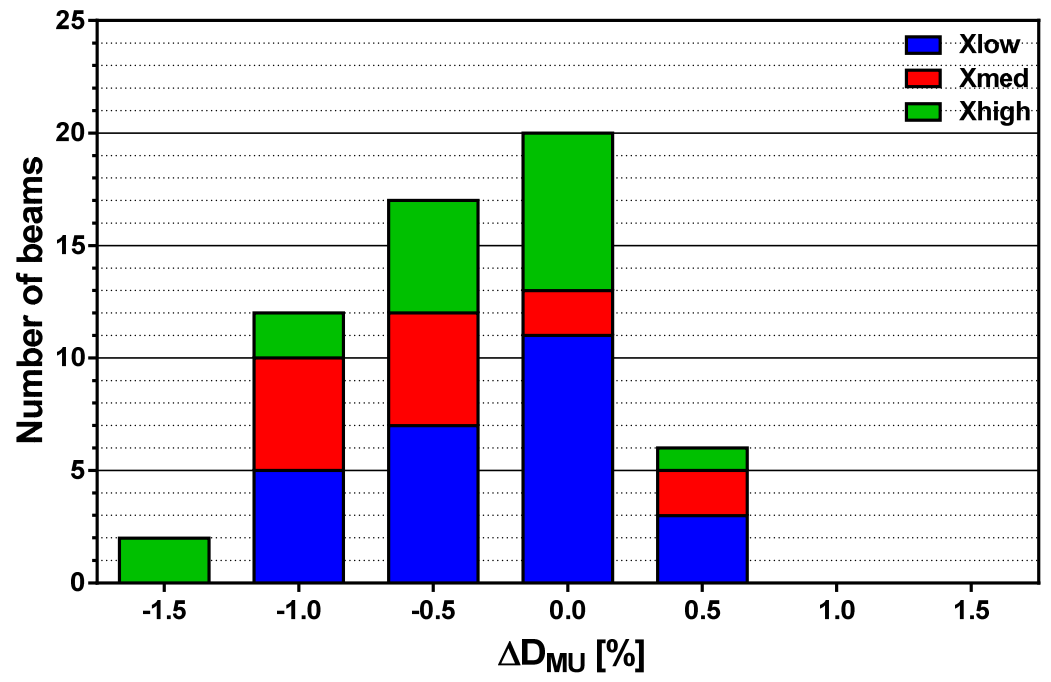

Figure 6: Results for the absolute dose measurements showing the distribution of the relative difference of $D_{\mathrm{W}}$ measurements performed by the institute, following their protocol and the $D_{\mathrm{W}}$ measurements performed by the Audit committee, for all 3 energy ranges, following NCS-18.

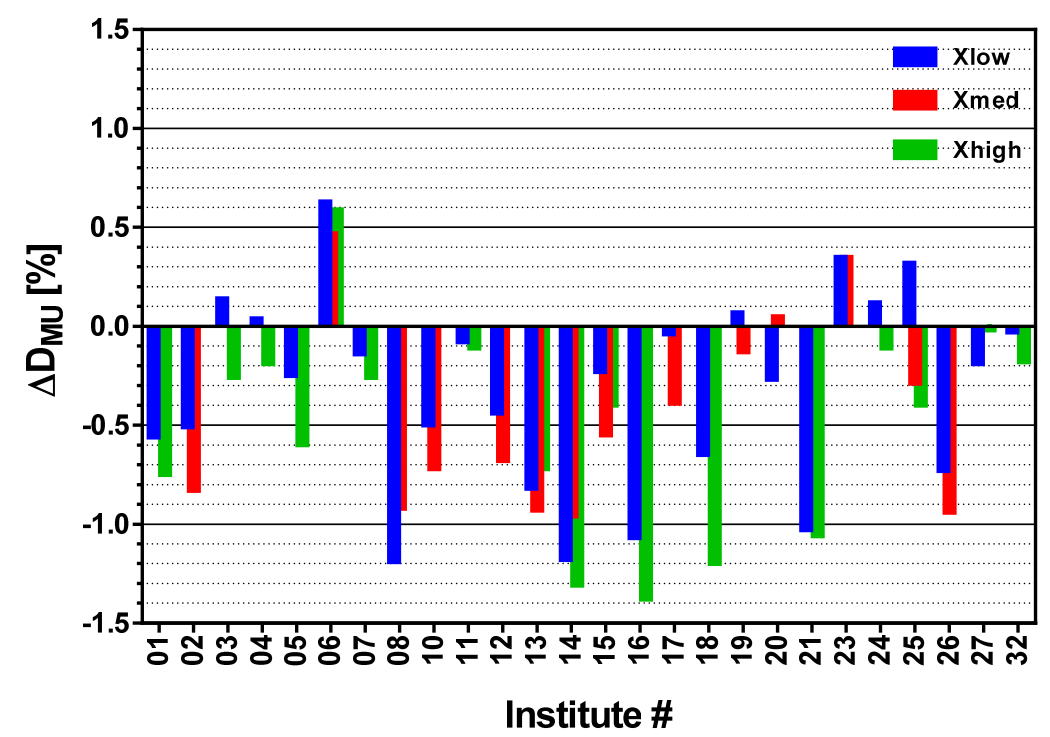

Figure 7: Results for the absolute dose measurements showing the relative difference of $D_{\mathrm{w}}$ measurements performed by the institute, following their protocol and the $D_{\mathrm{w}}$ measurements performed by the audit committee per institute for all 3 energy ranges, following NCS-18. 
Table 6: Results for the audit result, $\Delta D_{M U}$, performed by the institute and the $D_{\mathrm{w}}$ measurements performed by the Audit committee per institute for all 3 energy ranges.

\begin{tabular}{c|ccc}
\hline & $\mathbf{X}_{\text {low }}$ & $\mathbf{X}_{\text {med }}$ & $\mathbf{X}_{\text {high }}$ \\
\hline \# beams & 26 & 14 & 17 \\
Max & $-1.18 \%$ & $-0.95 \%$ & $-1.37 \%$ \\
Average & $-0.31 \%$ & $-0.46 \%$ & $-0.48 \%$ \\
SD & $0.48 \%$ & $0.48 \%$ & $0.52 \%$ \\
\hline
\end{tabular}

\subsubsection{Audit NCS-18 vs. Institute NCS-18}

Eighteen departments applied NCS-18; not every department used the NCS-18 in routine practice already.

Figure 8 shows the audit result, $\Delta D_{\mathrm{MU}}$, of the measured absorbed dose to water from the department to the measured absorbed dose to water by the audit team. Results are categorized by $T P R_{20,10}$ range as defined in Table 3.

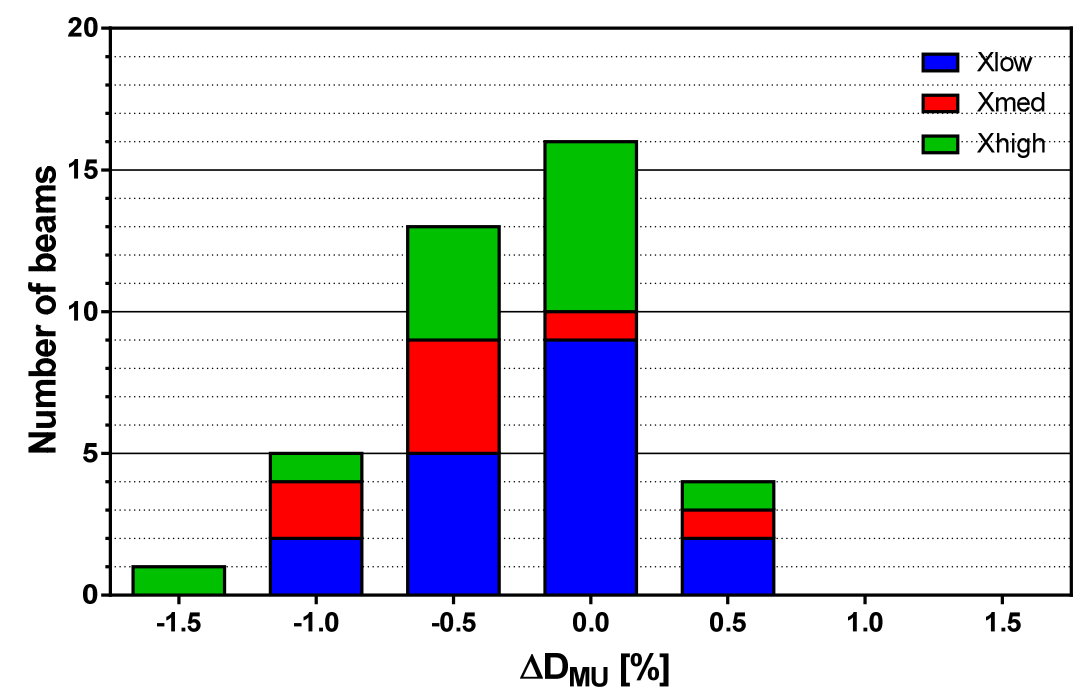

Figure 8: Results for the absolute dose measurements showing the distribution of the relative difference of $D_{\mathrm{w}}$ measurements performed by the institute and the $D_{\mathrm{w}}$ measurements performed by the audit committee, for all 3 energy ranges, both following NCS-18. 


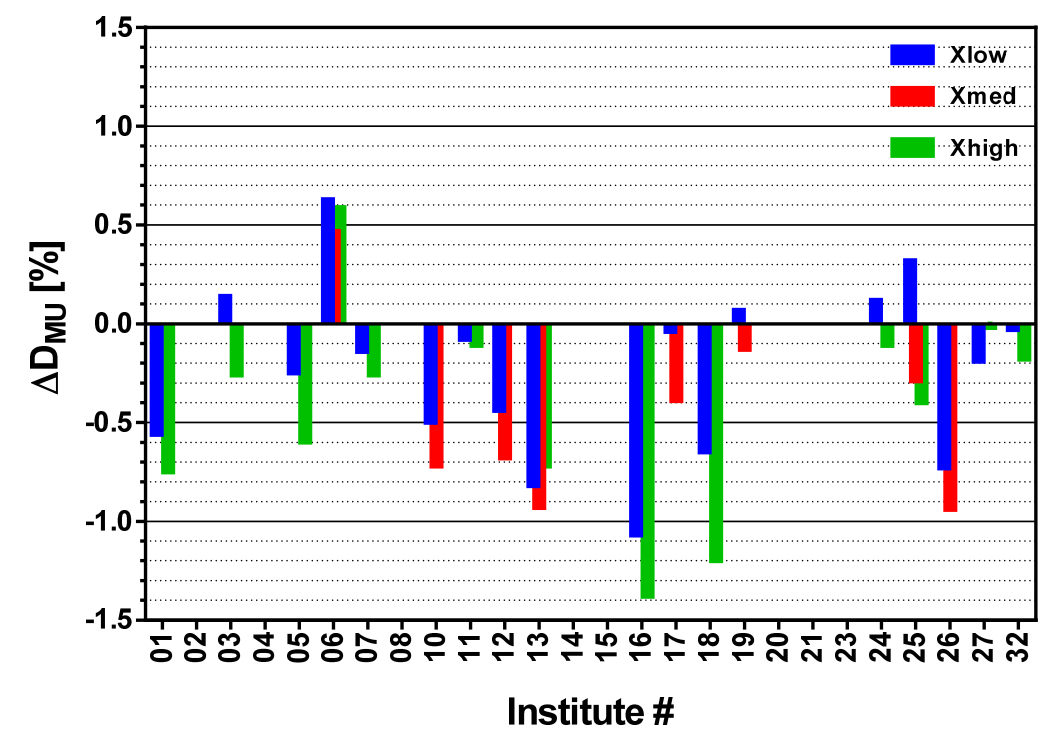

Figure 9: Results for the absolute dose measurements, showing the relative difference of $D_{\mathrm{w}}$ measurements performed by the institute and the $D_{\mathrm{w}}$ measurements performed by the Audit committee per institute for all 3 energy ranges, both following NCS-18.

Table 7: Results for the relative differences of $D_{w}$ performed by the institute and the $D_{w}$ measurements performed by the Audit committee per institute for all 3 energy ranges.

\begin{tabular}{c|ccc}
\hline & $\mathbf{X}_{\text {low }}$ & $\mathbf{X}_{\text {med }}$ & $\mathbf{X}_{\text {high }}$ \\
\hline \# beams & 18 & 8 & 13 \\
Max & $-1.06 \%$ & $-0.93 \%$ & $-1.37 \%$ \\
Avg. & $-0.23 \%$ & $-0.45 \%$ & $-0.41 \%$ \\
SD & $0.43 \%$ & $0.47 \%$ & $0.52 \%$ \\
\hline
\end{tabular}

\subsubsection{Audit NCS-18 vs. Institute NCS-2}

Eight departments did not apply the NCS-18.

Figure 10 shows the audit result, $\Delta D_{\mathrm{MU}}$, of the measured absorbed dose to water from the department to the measured absorbed dose to water by the audit team. Results are categorized by $T P R_{20,10}$ range as defined in Table 3 . 


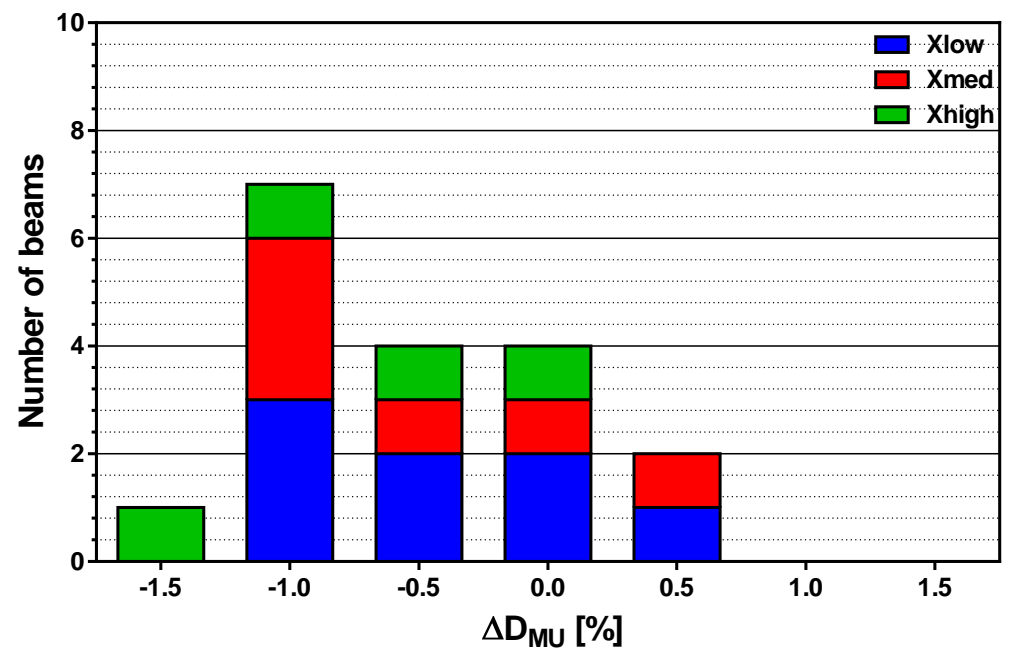

Figure 10: Results for the absolute dose measurements showing the distribution of the relative difference of NCS-2 $D_{\mathrm{w}}$ measurements performed by the institute and the NCS-18 $D_{\mathrm{w}}$ measurements performed by the Audit committee per institute for all 3 energy ranges.

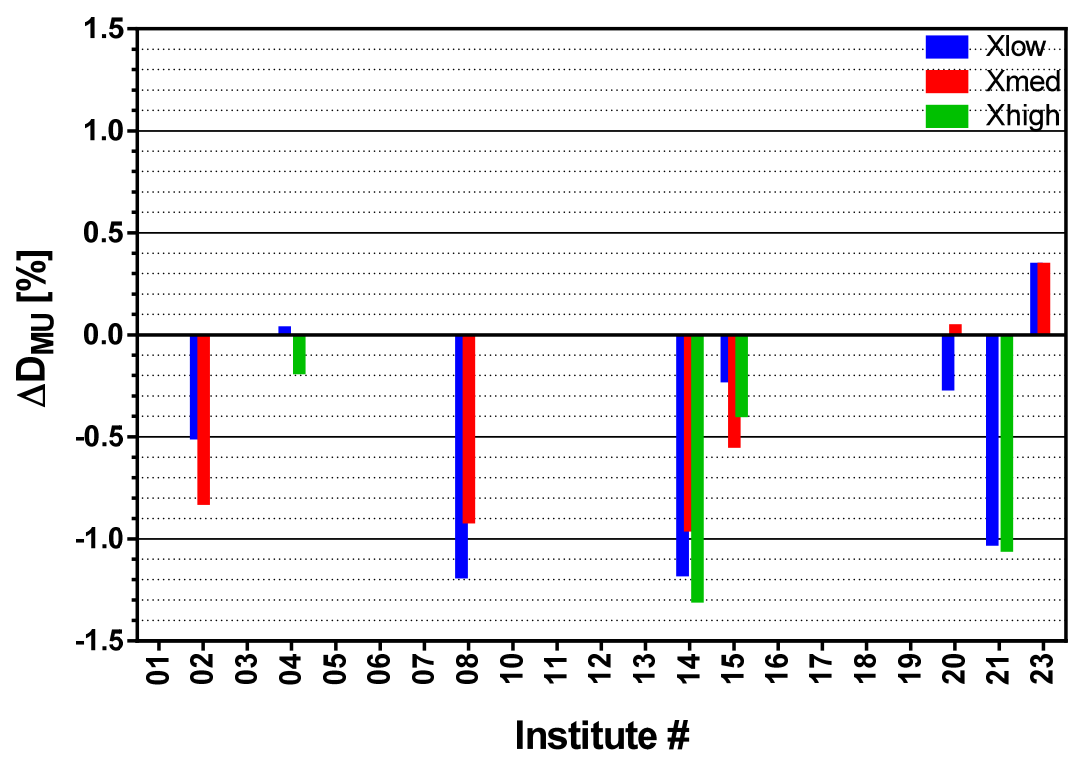

Figure 11: Results for the absolute dose measurements showing the relative difference of NCS-2 $D_{\mathrm{w}}$ measurements performed by the institute and the NCS-18 $D_{\mathrm{W}}$ measurements performed by the Audit committee per institute for all 3 energy ranges.

\subsubsection{Audit NCS-18 vs. Audit NCS-2}

The measurements of the absolute dose to water, $D_{\mathrm{W}, \mathrm{NCS}}$, were also calculated for NCS-2 protocol. Since the main focus of this audit was on the inter-department comparison of the 
new NCS-18 CoP, the results in this section are only given as average and standard deviation (Table 8).

Table 8: Statistics for the relative differences of $D_{w}$ performed by the Audit committee, following NCS-2 and following NCS-18, for all 3 energy ranges.

\begin{tabular}{c|ccc}
\hline & $\mathbf{X}_{\text {low }}$ & $\mathbf{X}_{\text {med }}$ & $\mathbf{X}_{\text {high }}$ \\
\hline \# beams & 26 & 14 & 17 \\
Max & $-0.51 \%$ & $-0.24 \%$ & $-0.20 \%$ \\
Avg. & $-0.36 \%$ & $-0.19 \%$ & $-0.16 \%$ \\
SD & $0.04 \%$ & $0.02 \%$ & $0.02 \%$ \\
\hline
\end{tabular}

\subsubsection{Ambient factors}

Only a few institutes have a traceable calibration for the measurement of temperature and air pressure. It was decided not to analyse differences in the absolute dose as a result of the measurement of temperature and pressure. 


\section{Discussion}

\subsection{TPR 20,10 comparison}

The results of the $T P R_{20,10}$ comparisons between the institutes and the NCS show average deviations at $\mathrm{X}_{\text {low }}, \mathrm{X}_{\text {med }}$ and $\mathrm{X}_{\text {high }}$ of respectively $0.26 \%, 0.20 \%$ and $0.18 \%$, determined with statistical (type A) uncertainties, originating from their respective standard deviation of the mean of about $0.20 \%(k=2)$. The overall (type $A$ and type $B$ ) expanded uncertainty, $U$, determined for the NCS TPR 20,10 measurement is $0.50 \%(k=2)$. Applying the statistical $E_{n}$ test on the comparison, where the result of $E_{n}$ should be smaller than 1 (95\% confidence) in order for agreement between the measurements to be confirmed:

$$
E_{n}=\frac{\left|\left(T P R_{20,10}\right)_{i}-\left(T P R_{20,10}\right)_{N C S}\right|}{\sqrt{\left(U_{T P R, i}\right)^{2}+\left(U_{T P R, N C S}\right)^{2}}}
$$

Results in $E_{n}$ values at $X_{\text {low }}, X_{\text {med }}$ and $X_{\text {high }}$ of respectively $0.47,0.36$ and 0.33 which confirm that for three energy ranges, with respect to the average results, no significant difference between the institute measurements and the NCS measurement can be identified.

The maximum deviation between the $T P R_{20,10}$ measurement of an individual institute compared to the NCS $\operatorname{TPR}_{20,10}$ value is $1.2 \%$ which would propagate via $\mathrm{k}_{\mathrm{Q}, \mathrm{Q}}$, (sensitivity coefficient $=0.32$ ) to a maximum deviation in the measured absorbed dose determination of $0.33 \%$ (see Table 5).

Furthermore, the deviation in $T P R_{20,10}$ for the different energies within a certain institute was small, indicating that consistent measurements were performed. The deviation between the institute and the audit might be caused by differences in measurement devices and setup. For example, the NCS audit team used a phantom that has less scattering material 'behind' the chamber when measuring at larger depth $(20 \mathrm{~cm})$. This might lead to less scatter at the respective higher depth, leading to a systematically lower value of the NCS TPR 20,10 value. Additionally, the institute's $T P R_{20,10}$ value was measured prior to the audit and is usually not measured on the actual audit day. 


\subsection{Dose to water comparison}

The results of the $D_{M U, w}$ comparisons between the institutes and the NCS show average deviations at $\mathrm{X}_{\text {low }}, \mathrm{X}_{\text {med }}$ and $\mathrm{X}_{\text {high }}$ of respectively $-0.31 \%,-0.46 \%$ and $-0.48 \%$, determined with statistical (type A) uncertainties, originating from their respective standard deviation of the mean $0.24 \%(k=2)$. The overall (type $A$ and type $B$ ) expanded uncertainty, $U$, determined for the NCS $D_{M U, N C S}$ measurement is $1.6 \%(k=2)$. Applying the statistical $E_{n}$ test on the comparison, where the result of $E_{n}$ should be smaller than 1 (95\% confidence) in order for agreement between the measurements to be confirmed:

$E_{n}=\frac{\left|\left(D_{M U}\right)_{i}-\left(D_{M U}\right)_{N C S}\right|}{\sqrt{\left(U_{M U, i}\right)^{2}+\left(U_{M U, N C S}\right)^{2}}}$

Results of $E_{n}$ values at $X_{\text {low }}, X_{\text {med }}$ and $X_{\text {high }}$ of respectively $0.20,0.29$ and 0.30 confirm excellent agreement between the institute's dose to water and that of the NCS. In other words: for the three energy ranges with respect to the average results, no significant difference between the institute measurements and the NCS measurement can be identified. However, Figure 7 and Table 6 suggest a small but significant systematic difference between the institutes' measurements and that of the NCS. In order to identify this difference, a closer look has to be taken at the NCS uncertainty budget, Table 1.

Since the NCS audit equipment and most of the institutes' dosimetry equipment was traceable to VSL, the uncertainty contribution of the calibration of the audit equipment would drop out of the NCS uncertainty budget. Its remaining uncertainty is expected to represent the comparison result based on an identical traceability.

The remaining NCS uncertainty budget then becomes $1.14 \%(k=2)$, resulting in $E_{n}$ values at $X_{\text {low }}, X_{\text {med }}$ and $X_{\text {high }}$ of respectively $0.46,0.41$ and 0.42 , which indicates that there is no statistical significant difference between the audit and institute's absorbed dose values.

The maximum deviation between the $D_{\mathrm{MU}, \mathrm{i}}$ and $D_{\mathrm{MU}, \mathrm{NCS}}$ amounted to $1.4 \%$ which is within the $k=2$ (95\% confidence) limit of the NCS uncertainty, regardless of the expected but undetermined uncertainty at the respective institute.

During the audit measurement, a deviation of $1.5 \%$ was determined as the action limit. The average deviation for all energy ranges is $-0.3 \%$.

The three different analysis categories provide similar results. 


\subsection{Equipment}

All institutes are capable of measuring absolute dose as stated in NCS-18 or NCS-2. However, between institutes a lot of variation in equipment exists. The main variations are within the type of water phantom size, chamber positioning and orientation, including the used water proof sheet and in the measurement of water temperature and ambient pressure.

Only a few institutes have a traceable calibration for the instruments which are used for the measurement of temperature and ambient pressure. Some institutes even use a website of a neighbouring weather station for the measurement of air pressure. The audit committee recommends these institutes to invest in instruments with a traceable calibration for the measurement of water (and/or air) temperature and ambient pressure. 


\section{Conclusions}

In total, 57 photon beams were audited in 26 institutes. For measurements based on NCS18, no deviations larger than $1.4 \%$ were found. No deviations were found in the implementation of the NCS-18 CoP in the participating institutes. In several occasions the results of this audit were used in international clinical trials (EORTC).

The NCS emphasizes the necessity of absolute dosimetry audits between (regional) institutes. This helps to ensure a good uniformity in absolute dose. Therefore, the NCS advises institutes to participate in absolute dosimetry audits on a periodic basis. This period may depend on national regulations or demands from clinical trials, but should not exceed 3 years.

Besides audits in absolute dosimetry, audits designed to meet the high standard of accuracy, necessary for the multi-segmented IMRT, VMAT or other modern treatments, are highly recommended. 


\section{Appendices}

\section{A. Working instructions}

The subcommittee has released their working instructions together with this report, so that others can benefit from these instructions.

The working instructions document can be downloaded from the NCS Website:

\section{www.radiationdosimetry.org}

\section{A.1 Use of working instructions}

The working instructions for the audit measurements are created with very high detail in respect to the equipment set-up used by the committee. The document can be used by audit committees or institutes as a guidebook to set up their own working instructions.

\section{A.2 Disclaimer}

These working instructions are provided for the convenience of the user for measuring absolute dosimetry for High-energy photon beams in radiotherapy using the NCS-18 Code of Practice. In creating these working instructions, every effort has been made to create conditions that minimize input errors. The NCS assumes no responsibility for user-calculated results, or for the impact of such upon dose determination. 


\section{Acknowledgements}

The subcommittee would like to thank PTW-Freiburg, Germany and VSL, Delft, the Netherlands for providing the equipment and chamber calibrations needed to perform all measurements.

During the audit the subcommittee members have met friendly and cooperative physicists in all participating institutes. This resulted in on-site visits, which were interactive and allowed for immediate comparison of various aspects.

We would like to thank everybody from the participating institutes who made the audit measurements possible. 


\section{References}

[1] NCS report \#18, Code of practice for the absorbed dose determination in high energy photon and electron beams, Delft, the Netherlands, 2008, www.radiationdosimetry.org

[2] NCS report \#2, Code of practice for the absorbed dose determination in high energy photon beams, Delft, the Netherlands, 1986, www.radiationdosimetry.org

[3] NCS report \#5, Code of practice for the absorbed dose determination in high energy electron beams, Delft, the Netherlands, 1989, www.radiationdosimetry.org

[4] M.Th. Hoornaert, J. Van Dam, S. Vynckier and A. Bouiller, A dosimetric quality audit of photon beams by the Belgian Hospital Physicist Association, Radiotherapy and Oncology 28 (1993) 37-43

[5] Wittkamper, F.W., Mijnheer, B.J. and van Kleffens, H.J. Dose intercomparison at the radiotherapy departments in the Netherlands. Part 1: Photon beams under reference conditions and for prostatic cancer treatment. Radiother. Oncol. 9 : 34-44, 1987.

[6] Kruijf, W.J.M., Regionale vergelijking absolute dosimetrie, Internal report, Medisch Centrum Haaglanden, locatie Westeinde, May 2005

[7] Piersma, H., van Haaren, P. and Nagel C., Absolute Dosimetrie Audit, Internal report, Cluster Noord-Holland, October 2007.

[8] Weinhous M.S., Meli A.M., Determinating Pion, the correction factor for recombination losses in an ionisation chamber, Med. Phys. 11 846-849, 1984.

[9] EA-4/02, Expression of the Uncertainty of Measurement in Calibration M:1999, European Cooperation for Accrediation

[10] Guide to the expression of uncertainty in measurement, JCGM 100:2008

[11] BIPM, Comité International des Poids et Mesures, Section I, Rayons $\mathbf{X}$ et $\gamma$, électrons (2-5 mat; 12-13 décembre), 4e réunion - 1977 
https://doi.org/10.25030/ncs-023 The NCS report has been downloaded on 26 Apr 2023 